\title{
Optimizing Long-Term Bank Financial Products Portfolio Problems with a Multiobjective Evolutionary Approach
}

\author{
Jian Xiong $\mathbb{D}^{1},{ }^{1}$ Chao Zhang, ${ }^{2}$ Gang Kou $\mathbb{D}^{1},{ }^{1}$ Rui Wang $\left(\mathbb{D},{ }^{3}\right.$ Hisao Ishibuchi, ${ }^{4}$ \\ and Fawaz E. Alsaadi ${ }^{5}$ \\ ${ }^{1}$ School of Business Administration, Southwestern University of Finance and Economics, Chengdu 610074, China \\ ${ }^{2}$ School of Economics, Southwestern University of Finance and Economics, Chengdu 610074, China \\ ${ }^{3}$ College of Systems Engineering, National University of Defense Technology, Changsha 410073, China \\ ${ }^{4}$ Department of Computer Science and Engineering, Southern University of Science and Technology, Shenzhen 518055, China \\ ${ }^{5}$ Department of Information Technology, Faculty of Computing and IT, King Abdulaziz University, Jeddah, Saudi Arabia
}

Correspondence should be addressed to Jian Xiong; xiongjian2017@swufe.edu.cn and Rui Wang; ruiwangnudt@gmail.com

Received 7 August 2019; Revised 9 February 2020; Accepted 28 February 2020; Published 7 April 2020

Academic Editor: Átila Bueno

Copyright ( $\odot 2020$ Jian Xiong et al. This is an open access article distributed under the Creative Commons Attribution License, which permits unrestricted use, distribution, and reproduction in any medium, provided the original work is properly cited.

\begin{abstract}
With the development of economy, the requirement of financial planning for individuals or families is emerging. In the era of the Internet, individual investors can conveniently enter the market and purchase financial products. Traditional portfolio management models focus on risky markets such as stock markets. However, risk-averse investors, such as normal families, may concern appropriate long-term financial planning. This paper considers the problem of portfolio management of bank financial products with a long-term planning horizon. By taking into account the final return and the flexibility, a multiobjective model of long-term portfolio is proposed. A multiobjective evolutionary approach is employed for the handling of conflicting objectives. Test instances are generated to illustrate the problem. Experiment results show that the presented algorithm can efficiently find trade-off solutions. Our experimental results also show that crossover probabilities should be separately implemented for longterm portfolio problems with hybrid encoding. Performance comparison of different crossover operators suggest that, for a realvalued encoding part, the simulated binary crossover (SBX) has a better performance than BLX- operator. While for a binary encoding part, a uniform crossover operator might be appropriate for large-scale instances. The proposed multiobjective model in this paper provides risk-averse investors with an appropriate decision support model for the long-term financial planning and management.
\end{abstract}

\section{Introduction}

Research studies on portfolio problems have gained much attention since the 1950s. Usually, portfolio return and risk are simultaneously considered as objectives to be optimized. By formulating risk measures from different perspectives, various models have been proposed in the literature, such as Mean-Variance (M-V) model [1], Mean-Semivariance (M-SV) model [2], Mean-Value at Risk (M-VaR) model [3], and Mean-Mean Absolute Deviation (M-MAD) model [4]. These models are widely employed for portfolio management in high-risk markets such as stock markets [5-7].
In the era of the Internet, the financial market becomes more diversified. Specifically, individual investors can conveniently enter the market. Moreover, various financial products are emerging with the development of the Internet and information technologies $[8,9]$. For instance, in China, almost each bank releases financial products via webs or mobile applications. Customers can purchase financial products anywhere and anytime. Compared to stock or fund products, financial products released by banks have lower risks. Many risk-averse investors prefer to choose such types of financial products. For example, most Chinese families tend to save their idle capital in the bank. In such a case, risk might not be the major concern of these risk-averse 
customers. However, when customers make their long-term financial planning, they still need to decide the type and the amount of products to be purchased, as well as the amount of capital should be reserved. In contrary, investors should manage the balance between the final return and the flexibility in the long-term financial planning.

Compared to traditional portfolio problems, models for a low-risk market with long-term planning horizon are rather scant. In this paper, we address the problem of longterm portfolio management with bank financial products. Based on real data of Chinese banks, this paper proposes a new portfolio model in which the final return and the flexibility are simultaneously optimized. In the proposed model, consideration of multiperiod investment decisions and realistic constraints such as the buy-in threshold increases the complexity of the problem. A multiobjective evolutionary algorithm (MOEA) is presented to solve the problem.

The contribution of the paper is twofold. Firstly, a new multiobjective model of portfolio is proposed. With consideration of realistic requirement of risk-averse investors, flexibility is designed as one of the objectives. The proposed model expands the scope of classic portfolio problems and can provide potential customers in financial markets with effective decision support model. Secondly, an effective algorithm is presented to solve the problem. The performance of different crossovers is investigated. A set of test instances are generated to illustrate the addressed problem and validate the effectiveness of the algorithm.

The remainder of this paper is organized as follows. Section 2 gives a brief review of the related work. A problem description and formulation are presented in Section 3. The solution technique is described in Section 4. Experimental results on generated test instances are reported in Section 5. Finally, summary and some conclusions are provided in Section 6.

\section{Related Work}

Various practical models of portfolio have been reported in the existing literature, such as uncertain portfolio selection problems [10-12]. The methodology of uncertain portfolio can be used for other practical problems such as multiobjective multi-item fixed charge solid transportation problem [13]. Practical portfolio problems are usually characterized by nonconvex and discrete objectives as well as constraints. Thus, metaheuristic algorithm such as evolutionary computation (EC) has become a popular solution technique for realistic portfolio problems. Among the first attempts, Arnone et al. [14] suggested the possibility of applying genetic approach to solve portfolio problems and presented some ideas. Maringer and Kellerer [15] developed a hybrid local search algorithm for cardinality constrained portfolios. Streichert et al. [16, 17] compared different crossovers and chromosome representations for constrained portfolio selection problems. Cura [18] presented a particle swarm optimization approach to solve portfolio optimization problems. Pai and Michel [19] studied portfolio problems with different types of constraints. The problems were converted to a single-objective optimization problem with an aggregation parameter. The proposed approach employed $k$-means cluster analysis to handle the cardinality constraint and used an evolution strategy to serve as an optimizer. Ruiz-Torrubiano and Suárez [20] proposed a memetic algorithm combining a genetic algorithm and quadratic programming for solving portfolio problems with cardinality constraints and transaction costs. Guo et al. [21] addressed a fuzzy multiperiod portfolio selection problem in which assets have different investment horizons. The authors designed a simulation-based genetic algorithm to solve the problem with different objectives, i.e., maximizing mean total return and minimizing variance.

Portfolio problems inherently have the trait of multiobjective optimization. The last two decades have witnessed the growth of taking advantage of MOEAs for solving portfolio problems. Doerner et al. [22] developed a Pareto ant colony optimization approach to solve project portfolio problems with objectives of benefit and remaining resources. Branke et al. [23] proposed an envelope-based multiobjective evolutionary algorithm to solve portfolio selection problems with cardinality constraints and minimum buy-in thresholds. With the rapid emerging of related work, some surveys focused on solving portfolio problems by means of MOEAs [24, 25]. Mishra et al. [26] addressed a realistic portfolio asset selection problem with budget, floor, ceiling, and cardinality constraints. The authors proposed a nondominated sorting multiobjective particle swarm optimization to solve the problem and conducted comparative performance assessment of a set of MOEAs. Kar et al. [27] proposed a fuzzy portfolio selection model for which Sharp ratio (SR) and Value at Risk ratio (VR) were simultaneously optimized by means of a multiobjective cellular genetic algorithm. Liagkouras [28] presented a three-dimensional encoding in an MOEA for portfolio problems. The proposed encoding technique can keep the processing time invariant to the problem size.

Although portfolio optimization is a classic problem in the area of finance and operations research, studies focusing on long-term portfolio planning are rather scant. Multiperiod portfolio can be classified as a type of long-term portfolio. Woodside-Oriakhi et al. [29] considered the problem of rebalancing an existing financial portfolio with transaction costs. Meghwani and Thakur [30] proposed a triobjective portfolio optimization model with risk, return, and transaction costs. The authors adapted classic MOEAs for solving the problem. However, either in single-period or multiperiod portfolio problems, objectives are usually portfolio return and related risks. These models are appropriate for the stock market. To the best of our knowledge, there is no model to consider portfolio flexibility in longterm financial planning in a low-risk market.

\section{Problem Statement}

3.1. Real Data. In this section, we first present some real data of financial products released by some banks in China. Usually, according to whether a product period exists, bank financial products can be classified as two types: open-ended 
financial products and close-ended financial products. For the latter type of products, the capital allocated to a product cannot be redeemed until the period is ended. According to the data released by Agricultural Bank of China (ABC) on 07 May 2019 (http://ewealth.abchina.com/fs/filter/), there are 93 financial products available in the market. Most financial products provided by ABS belong to the close-ended type. We select 20 close-ended products and report the detail information in Table 1. We also report similar data from Bank of Communications (BC)(http://www.bankcomm. com/BankCommSite/default.shtml). Products released by $\mathrm{BC}$ include close-ended and open-ended types. We select 10 close-ended products and 10 open-ended products and report the information in Table 2.

One can see from the data that products released by banks have three main attributes: minimum buy-in amount, product period, and interest rate. According to the data of $\mathrm{ABC}$ and $\mathrm{BC}$, the product period can be modeled by a finite set $\{1,2,3,6,12\}$ with unit of month. It can also be seen from the abovementioned tables that interest rates of financial products can be fixed or floating. However, for products with floating interest rates, the floating ranges are rather small. Thus, all financial products can be considered as low risky or medium risky. When a risk-averse investor plans to purchase these bank financial products, she might not concern an immediate higher return of a product with a floating interest rate. What she most concerns is how to appropriately allocate her capital in the long run and obtain a high final return.

3.2. Basic Definitions. Based on the real data provided in Section 3.1, the formulation of the problem studied in this paper is refined. We consider an investment planning in a time horizon with $T$ periods. For the sake of simplicity, similar to Gutjahr et al. [31], we assume that each period indicates one month. There are $N$ financial products released by commercial banks. Each product has the following basic attributes:
(i) $s$ : buy-in threshold
(ii) $d$ : product period
(iii) $r$ : return rate per time unit

In this paper, we assume that return rate $r$ is deterministic and pregiven for each product. The new arrived capital of an investor at each time point is $C_{t}(t=1, \ldots, T)$. The total available capital at each time point is $B_{t}(t=1, \ldots, T)$. At each time point, the investor needs to allocate her capital to different products. Thus, the problem can be categorized as a multiperiod portfolio problem.

3.3. Example. In multiperiod portfolio problems, an investor reallocates her wealth among available products at the beginning of each period [32]. In the presence of the product period, we have to optimize the investment plan in the long run. We use a simple example to illustrate the problem. We suppose that there are three available products:
TABLE 1: Financial products released by Agricultural Bank of China.

\begin{tabular}{lcccc}
\hline No. & $\begin{array}{c}\text { Buy-in } \\
\text { threshold } \\
(10,000 \text { RMB })\end{array}$ & $\begin{array}{c}\text { Product } \\
\text { period } \\
\text { (day) }\end{array}$ & $\begin{array}{c}\text { Expected } \\
\text { return per } \\
\text { year }(\%)\end{array}$ & $\begin{array}{c}\text { Capital } \\
\text { guaranteed }\end{array}$ \\
\hline 1 & 0.2 & 360 & 2.85 & $\mathrm{~N}$ \\
2 & 0.2 & 181 & 2.05 & $\mathrm{~N}$ \\
3 & 1 & 62 & $3-4$ & $\mathrm{~N}$ \\
4 & 1 & 90 & $3.5-4.4$ & $\mathrm{~N}$ \\
5 & 1 & 90 & $2.5-5.2$ & $\mathrm{~N}$ \\
6 & 1 & 181 & $3-4.9$ & $\mathrm{~N}$ \\
7 & 1 & 181 & 4.15 & $\mathrm{~N}$ \\
8 & 5 & 187 & 4.1 & $\mathrm{~N}$ \\
9 & 5 & 244 & 4.1 & $\mathrm{~N}$ \\
10 & 5 & 252 & 4.15 & $\mathrm{~N}$ \\
11 & 5 & 279 & 4.3 & $\mathrm{~N}$ \\
12 & 10 & 90 & $2.5-5.9$ & $\mathrm{~N}$ \\
13 & 100 & 157 & 4.05 & $\mathrm{~N}$ \\
14 & 1 & 182 & $3.2-3.3$ & $\mathrm{Y}$ \\
15 & 1 & 365 & $3.4-3.5$ & $\mathrm{Y}$ \\
16 & 1 & 182 & $3.15-3.25$ & $\mathrm{Y}$ \\
17 & 1 & 34 & 3 & $\mathrm{Y}$ \\
18 & 1 & 62 & 3.1 & $\mathrm{Y}$ \\
19 & 1 & 181 & 3.25 & $\mathrm{Y}$ \\
20 & 2 & 182 & $3.45-3.55$ & $\mathrm{Y}$ \\
\hline
\end{tabular}

TABLE 2: Financial products released by bank of communications.

\begin{tabular}{lcccc}
\hline No. & $\begin{array}{c}\text { Buy-in } \\
\text { threshold } \\
(10,000 \text { RMB })\end{array}$ & $\begin{array}{c}\text { Product } \\
\text { period } \\
\text { (day) }\end{array}$ & $\begin{array}{c}\text { Expected } \\
\text { return per } \\
\text { year }(\%)\end{array}$ & $\begin{array}{c}\text { Capital } \\
\text { guaranteed }\end{array}$ \\
\hline 1 & 1 & 30 & 3.6 & $\mathrm{~N}$ \\
2 & 1 & 60 & 3.65 & $\mathrm{~N}$ \\
3 & 1 & 90 & 3.90 & $\mathrm{~N}$ \\
4 & 1 & 180 & 3.95 & $\mathrm{~N}$ \\
5 & 1 & 360 & 4.00 & $\mathrm{~N}$ \\
6 & 20 & 60 & 3.95 & $\mathrm{~N}$ \\
7 & 20 & 180 & 4.05 & $\mathrm{~N}$ \\
8 & 20 & 360 & 4.10 & $\mathrm{~N}$ \\
9 & 50 & 90 & 4.05 & $\mathrm{~N}$ \\
10 & 50 & 94 & 4.35 & $\mathrm{~N}$ \\
11 & 1 & N/A & 2.05 & $\mathrm{Y}$ \\
12 & 1 & N/A & 3.20 & $\mathrm{~N}$ \\
13 & 1 & N/A & 3.90 & $\mathrm{~N}$ \\
14 & 10 & N/A & 3.35 & $\mathrm{~N}$ \\
15 & 10 & N/A & 3.40 & $\mathrm{~N}$ \\
16 & 10 & N/A & 3.55 & $\mathrm{~N}$ \\
17 & 50 & N/A & 4.20 & $\mathrm{~N}$ \\
18 & 100 & N/A & 4.30 & $\mathrm{~N}$ \\
19 & 500 & N/A & 5.00 & $\mathrm{~N}$ \\
20 & 600 & N/A & 4.50 & $\mathrm{~N}$ \\
\hline
\end{tabular}

(i) Product $A$ is with a product period of 12 months. The buy-in threshold is 5 units and the return rate is $0.3 \%$ per period (one month).

(ii) Product $B$ is with a product period of 6 months. The buy-in threshold is 10 units and the return rate is $0.4 \%$ per period (one month).

(iii) Product $C$ is with a product period of 1 month. The buy-in threshold is 2 units and the return rate is $0.2 \%$ per period (one month). 
The investment horizon is 1 year, i.e., 12 periods. We suppose at the beginning of the first and the seventh period, there is available capital with an amount of 8 . If there is no period constraint for each product, the optimal investment plan can be shown, as in Figure 1. At each decision point, the investor can choose products with the highest interest rate if the buy-in threshold constraint is satisfied. In this plan, the final return with a compound rate at the end of the planning horizon is $\left(8 \times 1.003^{6}+8\right) \times 1.004^{6}=16.5365$.

Then, we consider the situation that the product period constraint is present. At the first decision point, if the investor makes the same decision as in Figure 1, i.e., investing all available budget on the product with the highest interest rate, the optimal investment plan given the first-period decision can be shown, as in Figure 2. At the seventh period, new budget is available. However, at this decision point, the cash invested on product $A$ cannot be redeemed because of the product period constraint. Thus, the best choice of the investor at the second decision point is product $C$. With this investment plan, the final return is $8 \times 1.003^{12}+$ $8 \times 1.002^{6}=16.3893$.

Clearly the abovementioned plan is not optimal. The optimal plan can be shown, as in Figure 3. The final return of the optimal plan is $6 \times 1.003^{12}+\left(2 \times 1.002^{6}+8\right) \times$ $1.004^{6}=16.4867$. For the plan shown in Figure 2 , all available capital is allocated to products with the highest return rates. However, this will cause inflexibility in the long run and result in possible loss of final return. Thus, in the long-term financial planning, it is worth considering the flexibility of a plan and making decisions with a global optimal perspective.

3.4. Multiobjective Model. In this section, we formally present the multiobjective model of the addressed long-term portfolio problems.

3.4.1. Decision Variables. At each time point $t$, the investor needs to decide:

(i) $u_{i}(t)$ : the capital amount allocated to product $i$, $i=1, \ldots, N$

\subsubsection{Constraints}

(i) Buy-in threshold constraint: this type of constraint is similar to a floor-ceiling constraint which imposes lower and/or upper bounds on the capital proportion allocated to each asset. In our problem, this constraint demands that if a product is selected, at least some amount $s_{i}$ should be bought, i.e., $\forall i, \forall t, u_{i}(t) \geq s_{i}$ if $u_{i}(t)$ is not zero.

(ii) Total available capital constraint: this constraint ensures that the sum of capital allocated to each product does not exceed the total available amount $B_{t}$ at each time point $t$. Then, the constraint can be formulated as $\sum_{i=1} u_{i}(t) \leq B_{t}$.

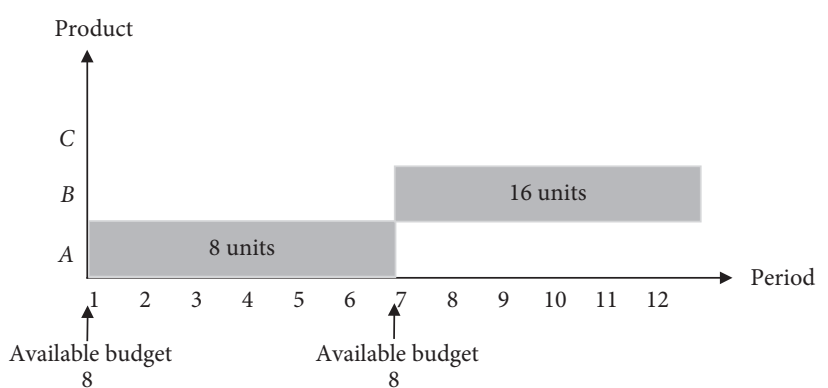

FIgURE 1: Optimal investment plan without product period constraint.

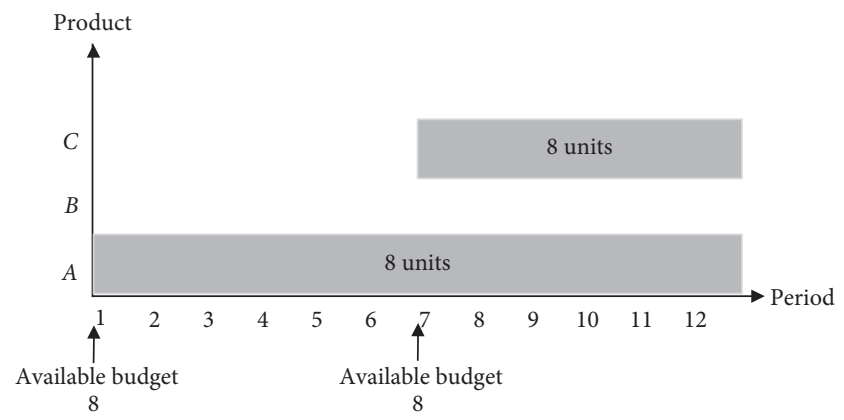

FIGURE 2: Investment plan with product period constraint and investing 8 units on product $A$ at the first period.

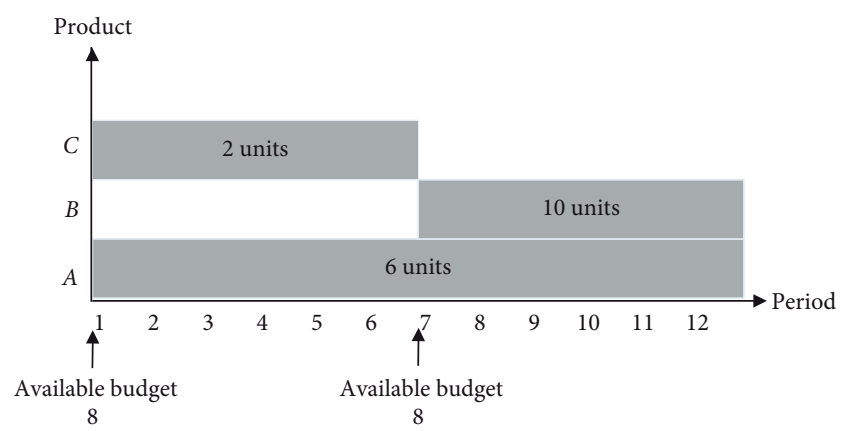

FIGURE 3: Optimal investment plan with product period constraint.

3.4.3. Objectives. For portfolio problems, the "maximizing return-minimizing risk" strategy is generally used as the definition of optimization objective(s) [25]. However, in this paper, we address the problem where the return rate of each product is deterministic. Then, the objective of minimizing risk is not the major task of the problem. Investors might pay much attention on the trade-off between the return and the flexibility of an investment plan. Thus, in our problem, maximizing return and minimizing inflexibility are the two objectives taken into account.

The portfolio return (PR) is calculated at the end of planning horizon. Recall that the planning horizon consists of $T$ periods. If an investor allocates the cash of $u_{i}$ to the $i$ th product at the beginning of the plan and it remains unchanged during the whole planning horizon, by the end of the $T$ period the product $i$ with the fixed return rate $r_{i}$ will be worth $u_{i}\left(1+r_{i}\right)^{T}$. However, investors may rebalance their 
investment at subsequent decision points according to the newly available capital. Then, the analytical formation of the portfolio return is difficult to present and the return can only be revealed at the end of the whole planning horizon.

Research studies on flexibility have gained much attention in many areas such as economics, organization, and manufacturing. Despite different definitions of flexibility with various backgrounds and perspectives, flexibility is usually used to measure the ability of dealing with future uncertainties. Some researchers measure the flexibility of capital investments by explicitly taking into account the cost of adopting these investments to possible changes in the future environment [33]. For the addressed problem, if an investor allocates all available capital on close-ended products with long periods, she can obtain a high final return at the end point of the planning horizon. However, there is no reserved capital until invested products expire. During this period, the investor is of weak ability to deal with uncertain events, such as configuring other household expenses. We consider such portfolio plans as inflexible. In other words, a higher final return of a portfolio will sacrifice the flexibility of the plan. Thus, the final return and the flexibility are in conflict with each other to certain extend. Then, an investor needs to balance between the final portfolio return and the reserved capital she holds during the planning horizon. Specifically, an investor tends to maximize the final return and minimize the inflexibility of a portfolio.

Before mathematically formulating the inflexibility measure, we first introduce a preference-based parameter $\underline{c}$ which indicates an investor's acceptable minimum level of holding the reserved capital. Let $e_{t}(t=1, \ldots, T)$ be the reserved capital at decision point $t$, i.e., capital which is not allocated to any financial products. If $e_{t}<\underline{c}$, it is considered as a risky situation. Then, an investment plan is considered as flexible if $e_{t} \geq \underline{c}$ for all $t$. In other words, the optimization task is to minimize a specific measure relating to risky points. Let $R$ be the set of risky reserved capital, i.e., $R=\left\{e_{t} \mid e_{t}<\underline{c}, t=1, \ldots, T\right\}$. The gap set is represented as $G=\left\{g_{t} \mid g_{t}=\underline{c}-e_{t}, e_{t} \in R\right\}$. The number of elements in $G$ is denoted as $|G|$. The mean and standard deviation of $G$ is, respectively, denoted as mean $(G)$ and $\operatorname{var}(G)$. Then, we formulate the inflexibility measure (IFM) of a portfolio by a modified mean - variance model as follows:

$$
\operatorname{IFM}=\{\alpha \times \operatorname{mean}(G)+(1-\alpha) \times \operatorname{var}(G)\} \exp \frac{|G|}{T},
$$

where $\alpha$ is a coefficient and is set to be 0.8 in this paper. From the abovementioned equation, one can see that the inflexibility measure corresponds to three parts: average gap to an investor's acceptable minimum level of holding reserved capital, variation of the gap, and the number of risky points.

It is clear that the inflexibility measure is to be minimized. For the sake of simplicity, we convert the objective of maximization of $P R$ to minimization of $-P R$ during the solving process. Then, objectives of the addressed problem can be presented as follows:

$$
\begin{aligned}
& \min f_{1}=-\mathrm{PR}, \\
& \min f_{2}=\mathrm{IFM} .
\end{aligned}
$$

\section{Methodology}

The addressed problem is combinatorial and the trade-off surface in the objective space might be nonconvex and disconnected. For such a type of problem, multiobjective evolutionary algorithms are preferable as solution techniques [34-37]. In this paper, we develop a solution technique based on a classic multiobjective genetic algorithm named NSGA-II [38], which is widely used to solve other financial problems such as feature selection in credit scoring [39]. In this paper, the chromosome and genetic operators are designed for our problem.

4.1. Chromosome Representation. For portfolio problems, genetic representation should include two types of information: selected product types and capital amount allocated to the selected products. Streichert et al. $[16,17]$ suggest to use a hybrid binary/real-valued encoding in which the binary part indicates whether an asset is included in the portfolio and the real-valued part is used to represent the weights allocated to individual assets. This type of representation is also employed by other approaches [23]. If there are $N$ products, the binary part and the real-valued part can be denoted as $\mathbf{k}=\left(k_{1}, \ldots, k_{N}\right)$ and $\mathbf{a}=\left(a_{1}, \ldots, a_{N}\right)$, respectively. Note that, for our problem, there are $T$ decision points. Thus, the dimension of the chromosome expands to $N \times T$. Then, we represent the chromosome by hybrid binary/real-valued matrices, as shown in Figure 4.

4.2. Genetic Operators. Crossover and mutation are two main operators for genetic algorithms. For the addressed problem, genetic operators are independently carried out on real-valued and binary matrix chromosomes.

Many types of crossover operators are designed for hybrid encoding of portfolio problems. However, comparative results of the effectiveness of different operators are rather scant. Two popular crossovers for real-valued chromosome are BLX- $\alpha$ and simulated binary crossover (SBX). Experimental results in Streichert et al. $[16,17]$ suggest that BLX-0.5 crossover performed best amongst three investigated recombination operators. In a recent work, SBX is used for crossing real-valued vectors [28]. In our experiments, we will analyze performances of these two different crossovers. The procedure of crossover for real-valued matrix is presented in Algorithm 1.

In classical portfolio problems, single-point crossover is usually employed to crossing binary vector [28, 40]. In our problem, crossover is carried out at each decision point, i.e., on each row of the binary matrix. Then, the procedure of single-point crossover is presented as in Algorithm 2.

Note that, in classic single-point crossover, two offspring are generated by swapping bits in the right part of the crossover point between two parents. For binary strings, swapping the right parts or the left parts of the parents does 


\begin{tabular}{|c|c|c|c|}
\hline$k_{11}$ & $k_{12}$ & $\cdots \cdots$ & $k_{1 N}$ \\
\hline$k_{21}$ & $k_{22}$ & $\cdots \cdots$ & $k_{2 N}$ \\
\hline$\cdots \cdots$ & $\cdots \cdots$ & $\cdots \cdots$ & $\cdots \cdots$ \\
\hline$k_{T 1}$ & $k_{T 2}$ & $\cdots \cdots$ & $k_{T N}$ \\
\hline
\end{tabular}

(a)

\begin{tabular}{|c|c|c|c|}
\hline$a_{11}$ & $a_{12}$ & $\cdots \cdots$ & $a_{1 N}$ \\
\hline$a_{21}$ & $a_{22}$ & $\cdots \cdots$ & $a_{2 N}$ \\
\hline$\cdots \cdots$ & $\cdots \cdots$ & $\cdots \cdots$ & $\cdots \cdots$ \\
\hline$a_{T 1}$ & $a_{T 2}$ & $\cdots \cdots$ & $a_{T N}$ \\
\hline
\end{tabular}

(b)

FIGURE 4: Matrix-based hybrid: (a) binary and (b) real-valued encoding.

(1) for $t=1$ to $T$ do

(2) for $n=1$ to $N$ do

(3) Generate rand from $[0,1]$

(4) if rand $\leq p c_{\text {real }}$ (crossover probability) then

(5) Apply BLX- $\alpha / \mathrm{SBX}$ crossover operator on element $a_{\mathrm{tn}}$

(6) end if

(7) end for

(8) end for

Algorithm 1: The procedure of crosssover operator for real-valued matrix.

(1) for $t=1$ to $T$ do

(2) Generate rand from $[0,1]$

(3) if rand $\leq p c_{\text {bin }}$ (crossover probability) then

(4) Randomly generate a crossover point $\mathrm{cp}_{1}$.

(5) Apply single-point crossover operator on the $t$ th row with $\mathrm{cp}_{1}$.

(6) end if

(7) end for

Algorithm 2: The procedure of single-point crossover for binary matrix.

not affect the generated offspring. However, in our problem, the chromosome is a binary matrix. Swapping the fixed parts of parents might generate biased children. Thus, a uniform crossover might be more appropriate for our problem. With a uniform crossover, offspring is obtained by randomly inheriting chromosome parts from two parents. In our experiments, we will compare the performances of singlepoint crossover and uniform crossover with a single crossover point, as well as two-point crossover and uniform crossover with two crossover points.

For the real-valued matrix, a widely used operator named polynomial mutation is employed for mutating the solution. The mutation operator for binary matrix is shown in Algorithm 3.

\section{Experimental Results}

5.1. Test Instances Generation. Compared to classic portfolios, the addressed problem has two different characteristics: (1) product might have different periods and (2) budget is available at each decision point. Thus, existing benchmark of portfolio cannot be used in our problem. Then, we randomly generated test instances to illustrate the problem and investigate the performance of the algorithm. In real cases, return rates $r$ usually depend on two parameters: product period $d$ and buy-in threshold $s$. From the real data reported in Section 3.1, for most cases, products with higher values of $d$ and $s$ usually have higher values of $r$. Then, in the test generation, we explicitly model the relation of parameters of $d, s$, and $r$ as follows:

$$
r=r_{0} \exp \left(\frac{d}{T}+\frac{s}{3 \widehat{s}}\right),
$$

where $r_{0}$ is baseline return rate and $\widehat{s}$ is the upper bound of the buy-in threshold for all products. We are aware of that this formulation might be different with real cases. However, in test instance generation, this will not affect the analysis process and related conclusive results. In future research, the relation between different parameters will be refined with more real data.

We generated two sets of instances, denoted as set ${ }_{1}$ and set $_{2}$. The set ${ }_{1}$ includes two instances, denoted as set $_{1}$ instance $_{1}$ and set $_{1}$ instance $_{2}$, with four products and a planning horizon of 24 periods. In both instances, in set ${ }_{1}$, parameters for the 4 products are the same, as shown in Table 3. The difference of the two instances lies in the 


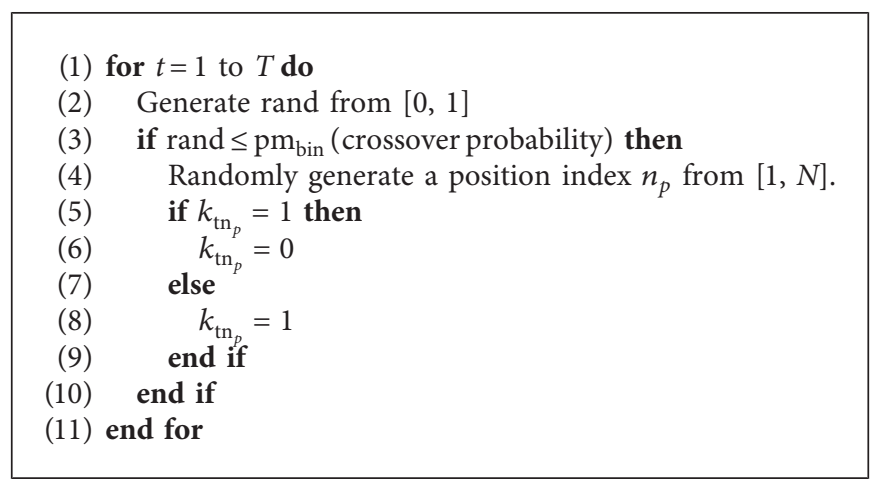

Algorithm 3: The procedure of mutation operator for binary matrix.

available capital set. For set $_{1-}$ instance $_{1}$, the set is $\{15,9,13,9,10,16,10,15,9,20,12,10,18,15,6,11,16,6,11$, $19,9,13,16,13\}$, while the set is $\{72,0,0,0,0,0,76,0,0,0,0$, $0,72,0,0,0,0,0,81,0,0,0,0,0\}$ for set $_{1}$ instance 2 . The frequency of capital arrival in set ${ }_{1}$ instance $_{2}$ is lower than that

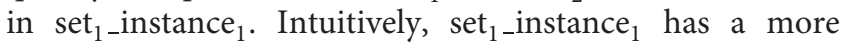
complex structure compared to set $_{1}$ instance $_{2}$.

The set $_{2}$ consists of six instances. Table 4 shows parameters in set $_{2}$. The product number varies from 10 to 35 . The planning period is 60 units. The other parameters are randomly drawn from intervals given in the table. For example, the parameter $C_{t}$ of instance 1 is randomly generated between the lower bound 1 and the upper bound 50 for each time unit.

5.2. Parameter Settings. For both instance sets, the population size was set as 200. The algorithm was terminated after 100 generations for set $_{1}$. Since set 2 has a larger size, the generation number was set as 200. As suggested in other studies $[16,17]$, crossover rates $\mathrm{pm}_{\text {real }}$ and $\mathrm{pm}_{\text {bin }}$ were set as 0.9 and the mutation rate was 0.1 . In our experiments, sensitivity of the performance of the algorithm on the crossover rates was analyzed on set $_{2}$ with a constant mutation rate 0.1 . In the calculation of hypervolume and IGD, objective values were normalized. For set $_{1}$, maximum and minimum values for horizontal axis were -200 and -800 and the values for vertical axis were 50 and 0 . These values for set $_{2}$ were set as -1000 and -3500 for horizontal axis and 60 and 0 for vertical axis, respectively. These values were determined with analysis of results obtained by a single run of the algorithm on set $_{1-}$ instance $_{1}$ and set $_{2}$ instance $_{1}$. All algorithms were repeated for 30 independent runs.

\subsection{Four-Product Examples}

5.3.1. Results of set _instance $_{1}$. We first plot the Pareto front of set ${ }_{1}$ instance ${ }_{1}$ with different $\underline{c}(\underline{c}=2,4,6,8,10)$, as shown in Figure 5. We plot portfolio return (PR) on the horizon axis and inflexibility measure (IFM) on the vertical axis. For each $\underline{c}$, the two endpoints of the Pareto front correspond to the portfolio plans with the maximum and the minimum averaged reserve capital. We list the two endpoints as well as the middle point of each Pareto front in Table 5.
TABLE 3: Parameters of products for the two instances of set $_{1}$.

\begin{tabular}{lccc}
\hline & $s$ & $d$ & $r_{0}$ \\
\hline Product 1 & 41 & 4 & 0.0399 \\
Product 2 & 10 & 2 & 0.0299 \\
Product 3 & 45 & 3 & 0.0393 \\
Product 4 & 50 & 4 & 0.0424 \\
\hline
\end{tabular}

TABLE 4: Parameters in the generation of set $_{2}$.

\begin{tabular}{|c|c|c|c|c|c|c|}
\hline & $\begin{array}{c}\text { Products } \\
\text { number } \\
(N)\end{array}$ & $T$ & $C_{t}$ & $s$ & $d$ & $r_{0}$ \\
\hline set $_{2-}$ instance $_{1}$ & 10 & 60 & {$[5,20]$} & {$[1,50]$} & {$[0,12]$} & $\begin{array}{l}{[0.0200,} \\
0.0300]\end{array}$ \\
\hline set $_{2}$ instance $_{2}$ & 15 & 60 & {$[5,20]$} & {$[1,50]$} & {$[0,12]$} & $\begin{array}{l}{[0.0200,} \\
0.0300]\end{array}$ \\
\hline set $_{2-}$ instance $_{3}$ & 20 & 60 & {$[5,20]$} & {$[1,50]$} & {$[0,12]$} & $\begin{array}{l}{[0.0200,} \\
0.0300]\end{array}$ \\
\hline set $_{2}$ instance $_{4}$ & 25 & 60 & {$[5,20]$} & {$[1,50]$} & {$[0,12]$} & $\begin{array}{l}{[0.0200,} \\
0.0300]\end{array}$ \\
\hline set $_{2-}$ instance $_{5}$ & 30 & 60 & {$[5,20]$} & {$[1,50]$} & {$[0,12$} & $\begin{array}{c}{[0.0200} \\
0.0300]\end{array}$ \\
\hline set $_{2}$ instance $_{6}$ & 35 & 60 & {$[5,20]$} & {$[1,50]$} & {$[0,12]$} & $\begin{array}{l}{[0.0200,} \\
0.0300]\end{array}$ \\
\hline
\end{tabular}

From Figure 5 and Table 5, one can see that the values of the maximum portfolio return are comparable despite different levels of $\underline{c}$. However, the value of IFM varies from 2.47 $(\underline{c}=2)$ to $12.45(\underline{c}=10)$. This is because the calculation of IFM depends on the predefined value of $\underline{c}$ while the return is only determined by investing structures of portfolios. Figure 6 shows investing structures of endpoints with the maximum return under different levels of $\underline{c}$ for set $_{1-i n s t a n c e}$. It can be seen that the structures of different endpoints with the maximum return are very similar.

Structures of endpoints with the minimum return under different levels of $\underline{c}$ for set ${ }_{1}$ instance $_{1}$ are shown in Figure 7. The main difference of portfolio structures in Figure 7 lies in the level of reserved capital at each decision point. Endpoints with the minimum return correspond to the portfolios in which the average reserved capital approaches to the pregiven level of $\underline{c}$. If $\underline{c}$ is set at a higher level, less capital is available for investing on products. Thus, the return of the 


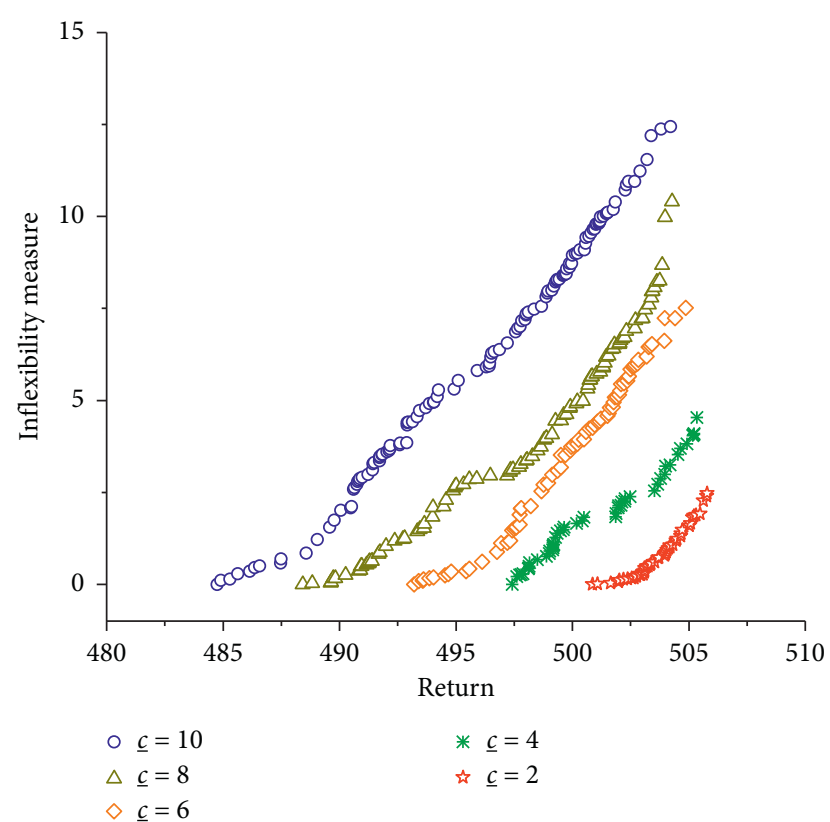

FIGURE 5: Obtained nondominated solutions with different $\underline{c}$ for set $_{1-\text { instance }}$. $_{\text {. }}$.

TABle 5: Two endpoints and the middle point with different $\underline{c}$ for set $_{1-}$ instance $_{1}$.

\begin{tabular}{lccc}
\hline & $\begin{array}{c}\text { Endpoint with } \\
\text { maximum return }\end{array}$ & $\begin{array}{c}\text { Endpoint with } \\
\text { minimum return }\end{array}$ & Middle point \\
\hline \multirow{c}{*}{$=2$} & $\mathrm{PR}=505.79$, & $\mathrm{PR}=500.86$, & $\mathrm{PR}=227.71$, \\
& $\mathrm{IFM}=2.47$ & $\mathrm{IFM}=0$ & $\mathrm{IFM}=1.27$ \\
$\underline{c}=4$ & $\mathrm{PR}=505.34$, & $\mathrm{PR}=497.42$, & $\mathrm{PR}=227.84$, \\
& $\mathrm{IFM}=4.53$ & $\mathrm{IFM}=0$ & $\mathrm{IFM}=4.38$ \\
$\underline{c}=6$ & $\mathrm{PR}=504.86$, & $\mathrm{PR}=493.21$, & $\mathrm{PR}=225.38$, \\
& $\mathrm{IFM}=7.51$ & $\mathrm{IFM}=0$ & $\mathrm{IFM}=5.03$ \\
$\underline{c}=8$ & $\mathrm{PR}=504.29$, & $\mathrm{PR}=488.43$, & $\mathrm{PR}=224.56$, \\
& $\mathrm{IFM}=10.41$ & $\mathrm{IFM}=0$ & $\mathrm{IFM}=7.35$ \\
$\underline{c}=10$ & $\mathrm{PR}=504.21$, & $\mathrm{PR}=484.75$, & $\mathrm{PR}=224.12$, \\
& $\mathrm{IFM}=12.45$ & $\mathrm{IFM}=0$ & $\mathrm{IFM}=10.54$ \\
\hline
\end{tabular}

corresponding portfolio will be lower compared to other portfolios with lower levels of $\underline{c}$. This can be seen in Figure 5 and Table 5, and returns of the endpoint with $\underline{c}=2$ is 224.17 while the return is only 203.35 for $\underline{c}=10$. From the perspective of decision making, an investor with a high level of $\underline{\underline{c}}$ can be considered as a risk-averse decision maker. While the investor with a low level of $\underline{c}$ is a risk-taking decision maker. It is reasonable that, for a risk-taking investor she might not accept portfolios with low return. While for a risk-averse investor, portfolios with low return but high level of flexibility will be acceptable. As shown in Figure 5, given limited computational resource, obtained nondominated solutions focus on the area of high returns for $\underline{c}=2$.

5.3.2. Results of set instance $_{2}$. We then plot the obtained approximate Pareto front of set ${ }_{1}$-instance ${ }_{2}$ with different $\underline{c}$ in Figure 8. One can see from Figure 8 that the shapes of the obtained approximate efficient fronts are closer to be linear, compared to the efficient fronts shown in Figure 5. The difference arises from the structure of the problem instances. For set ${ }_{1}$ instance 2 , a lower level of capital arrival frequency decreases the complexity of the problem and makes the investment policy simpler and clearer.

For set $_{1}$ instance $_{2}$, investing structures of obtained endpoints with maximum return under different levels of $\underline{c}$ are shown, as in Figure 9. We recall that, for set ${ }_{1}$ instance $_{2}$, there is sufficient available capital at each decision point. Thus, an investor can allocate all capital on one or more products with high return rates. Theoretically, endpoints with the maximum return correspond to portfolios in which the reserved capital at each point remains the level of 0 . This can be seen from Figure 9 that the level of reserved capital is rather flat and approaches to 0 .

5.3.3. Comparison of BLX and SBX Crossovers. In this section, we compare BLX- $\alpha$ and SBX operators on set ${ }_{1}$. As suggested in Streichert et al. [16,17], the value of $\alpha$ was set as 0.5 for BLX- $\alpha$ crossover. The value of $\underline{c}$ was set as 10 . Obtained nondominated solutions for set $_{1}$ instance $_{1}$ and set $_{1}$ instance $_{2}$ with different crossover operators are plotted in Figure 10. It is clear that SBX performed better than BLX0.5 for both instances. Note that, for set $_{1-}$ instance $_{1}$, the gap between nondominated solutions obtained with BLX-0.5 and SBX is larger than that of set $_{1}$ instance $_{2}$. As aforementioned, set $_{1}$ instance $_{2}$ is less complex than set $_{1}$ instance $_{2}$ and thus is easy to be solved. Since SBX outperforms BLX-0.5, we employed SBX as the crossover operator in the following experiments.

5.4. Results on set $_{2}$. In this section, we first investigated performances with different crossover probabilities. Then, performances with different crossover mechanism for binary matrix were compared. In the performance comparison, two most often used indicators named hypervolume [41] and Inverted Generational Distance (IGD) [42] were employed as performance measures. A larger value of hypervolume indicates a better performance and it is in contrast for IGD value. In the calculation of IGD, an approximating Pareto optimal set was obtained by merging all nondominating solutions in each run. Note that, for $\operatorname{set}_{2}$, the value of $\underline{c}$ was 30 for all instances.

5.4.1. Sensitivity Analysis of Crossover Probability. In this section, we provide sensitivity analysis of the crossover probability. As aforementioned, SBX crossover is employed for real-valued matrix. We first chose the single-point crossover for binary matrix in the analysis. The chromosome consists of real-valued and binary parts. Thus, it is worth investigating the sensitivity of the crossover probability for different parts. In previous research studies such as Liagkouras [28], the crossover probability is set identical for different chromosome parts. Firstly, we analyze the sensitivity of the crossover probability for the real-valued chromosome part. Figures 11 and 12 show the results of hypervolume and IGD with different levels of the crossover 


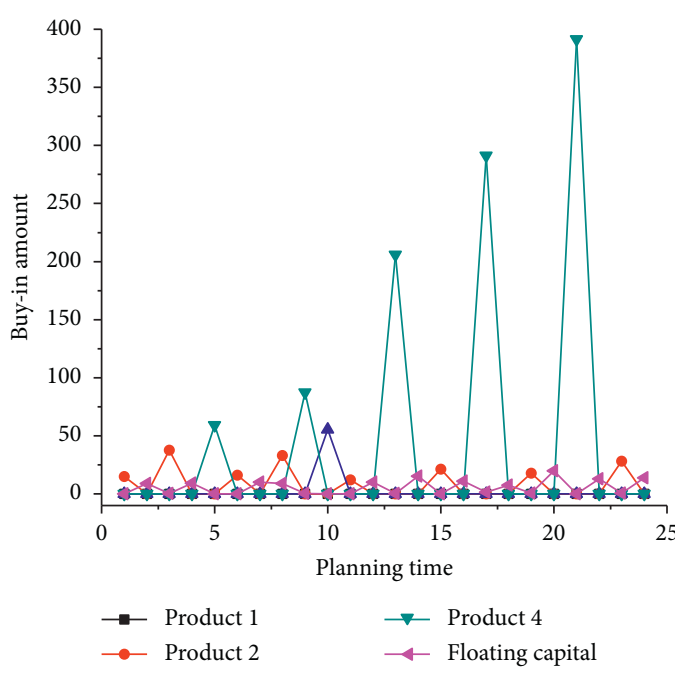

(a)

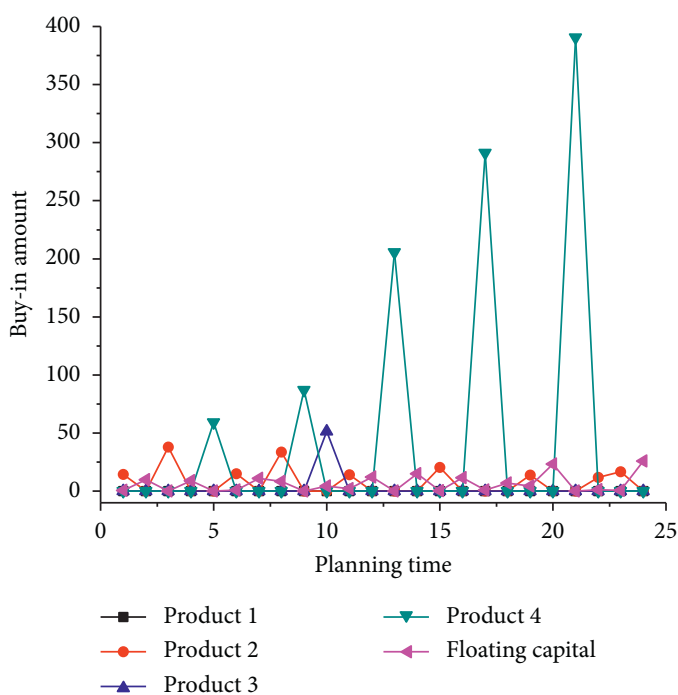

(c)

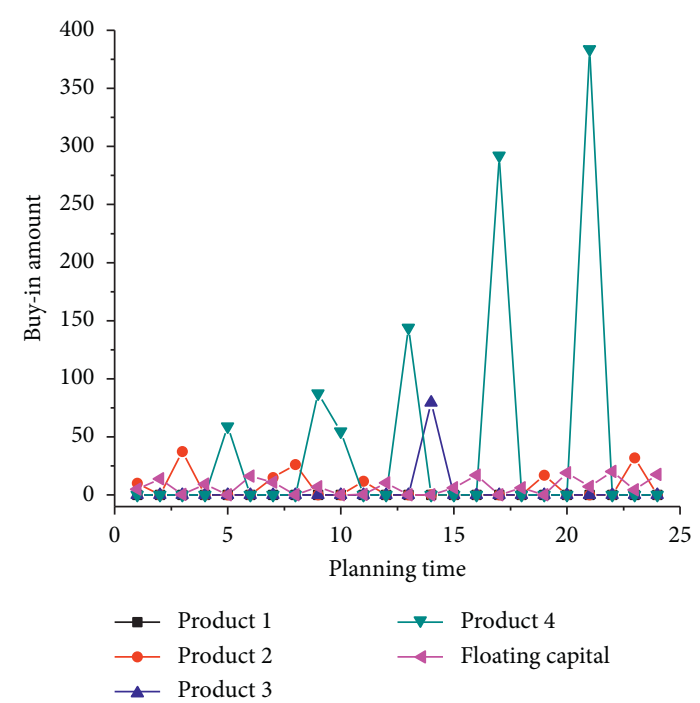

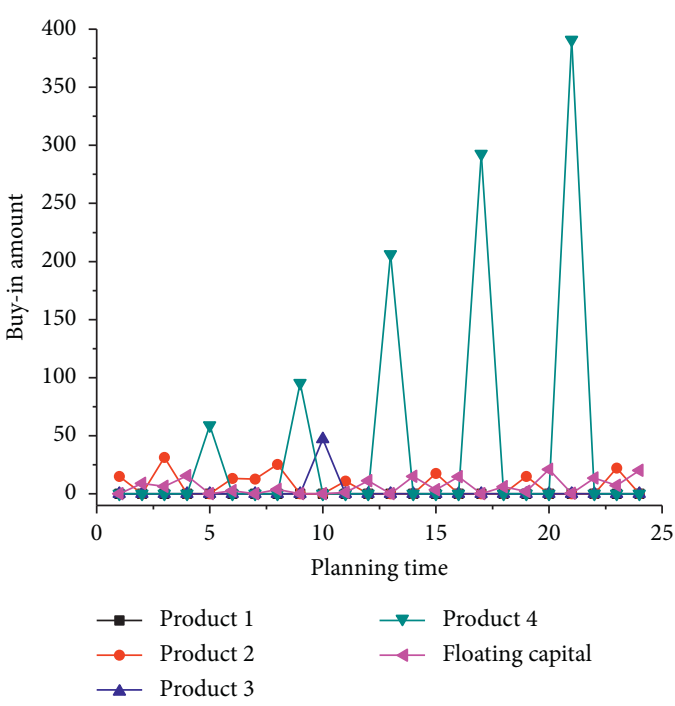

(b)

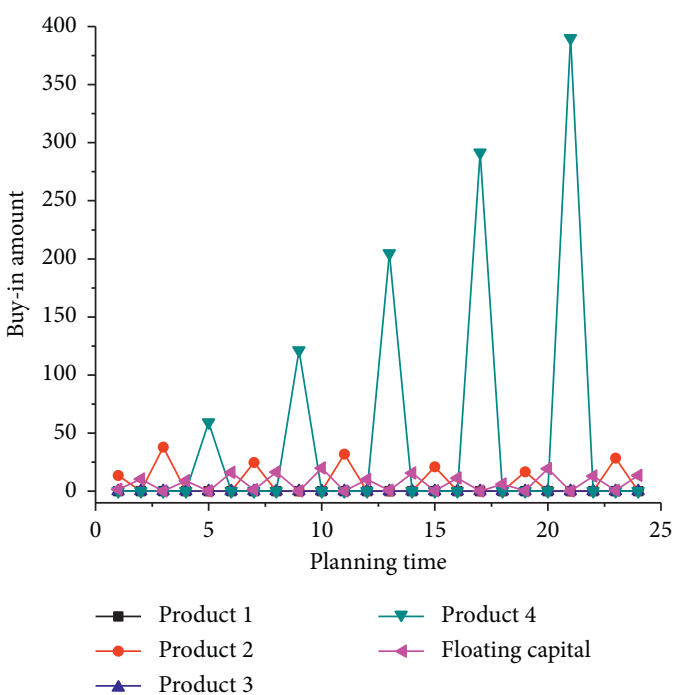

(d)

(e)

FIGURE 6: Structures of endpoints with maximum return with different $\underline{c}$ for set ${ }_{1}$ instance $_{1}$ : (a) $\underline{c}=2$, (b) $\underline{c}=4$, (c) $\underline{c}=6$, (d) $\underline{c}=8$, and (e) $c=10$. 


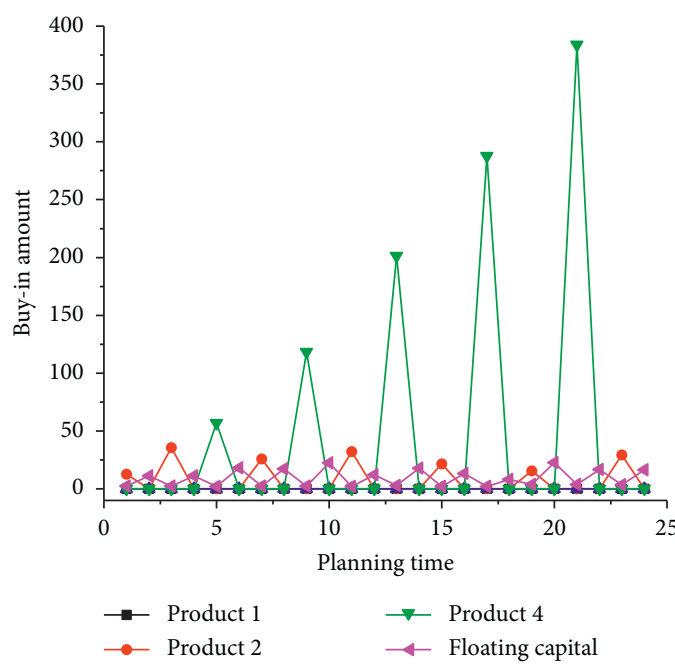

(a)

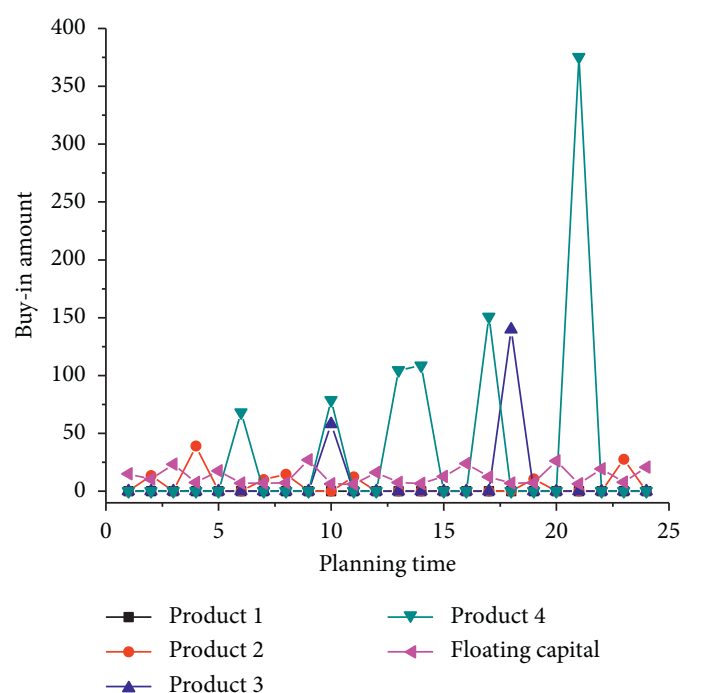

(c)

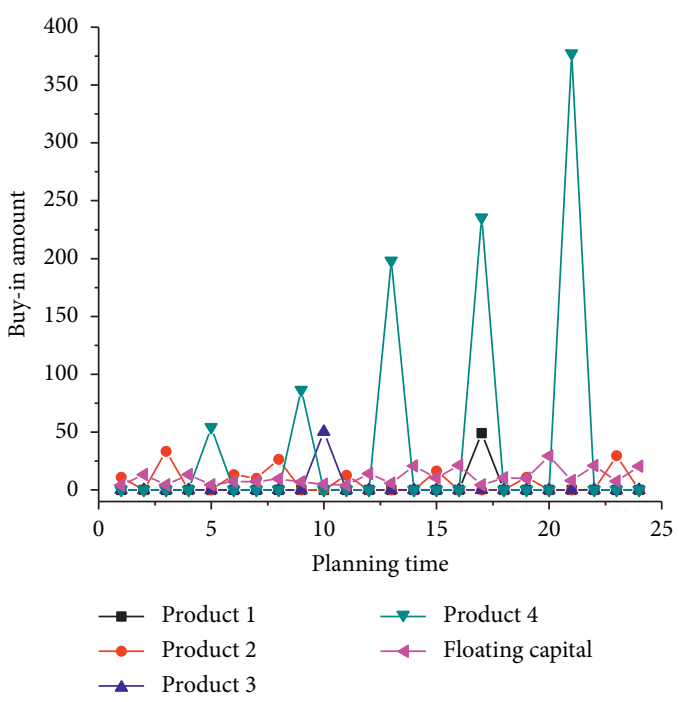

(b)

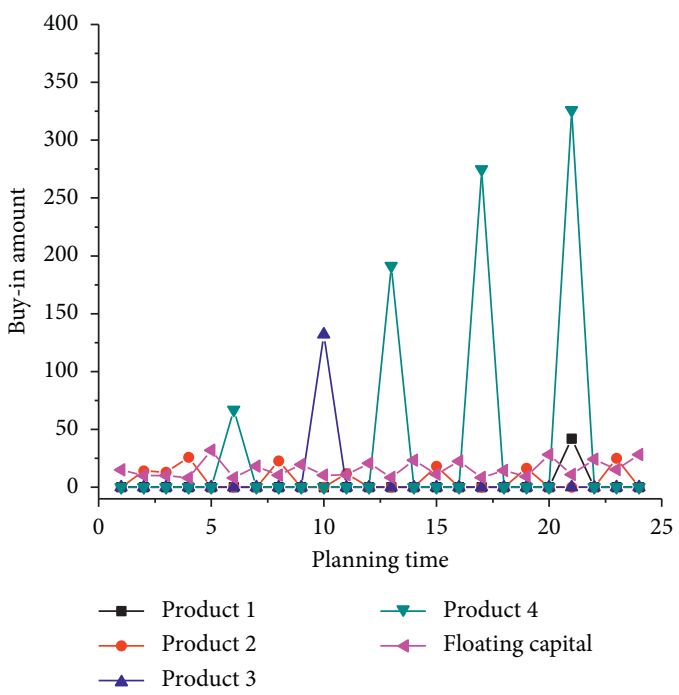

(d)

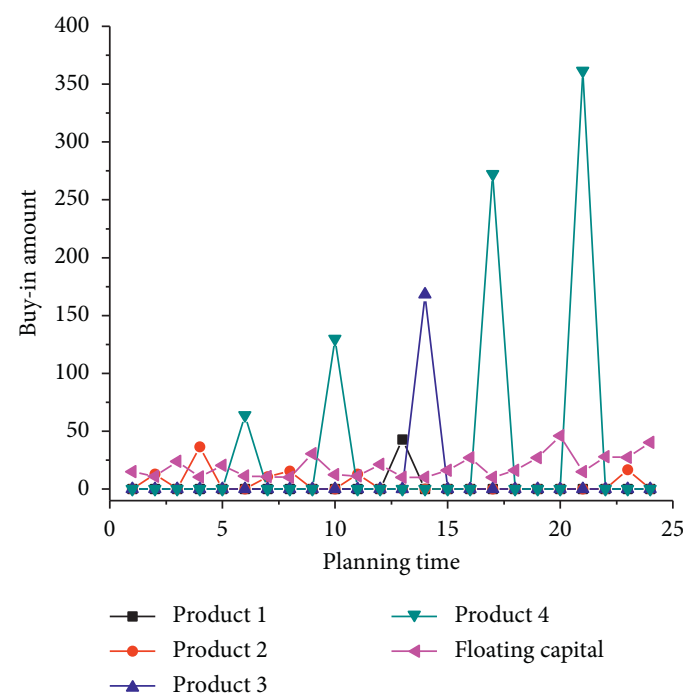

(e)

FIGURE 7: Structures of endpoints with minimum return with different $\underline{c}$ for set $_{1}$ instance ${ }_{1}$ : (a) $\underline{c}=2$, (b) $\underline{c}=4$, (c) $\underline{c}=6$, (d) $\underline{c}=8$, and (e) $\underline{c}=10$. 


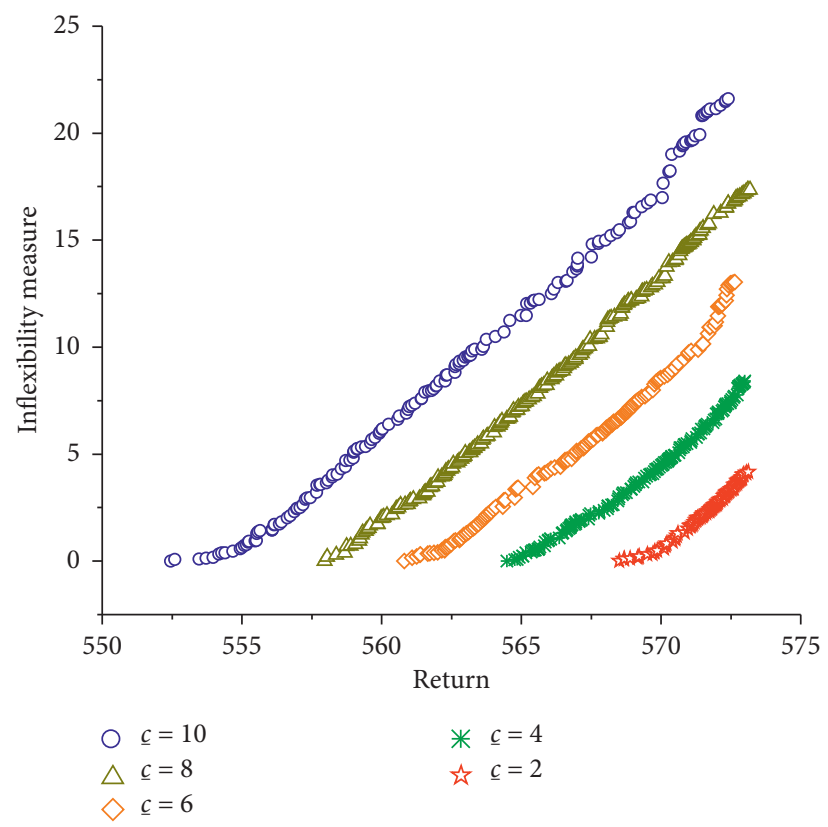

FIgURE 8: Obtained nondominated solutions with different $\underline{c}$ for set ${ }_{1}$ instance ${ }_{2}$.

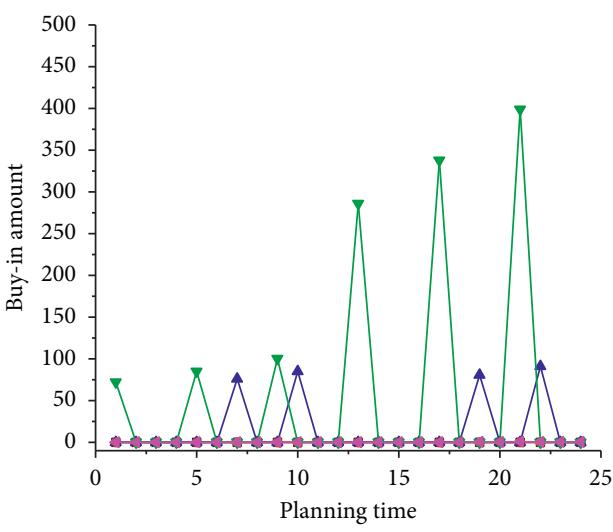

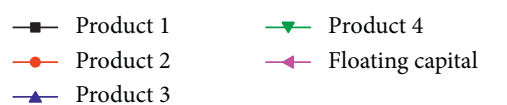

(a)
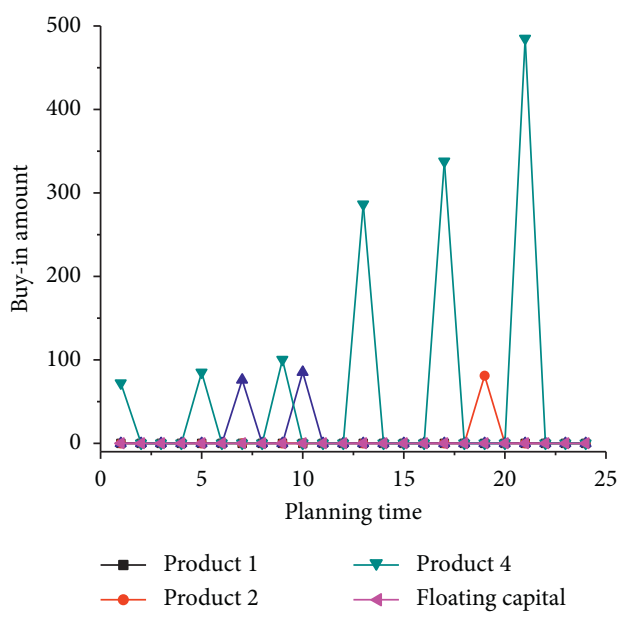

(c)

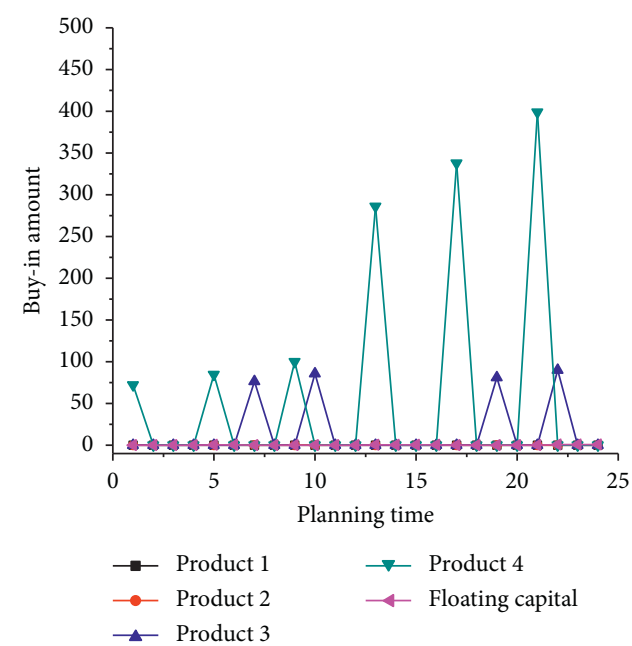

(b)

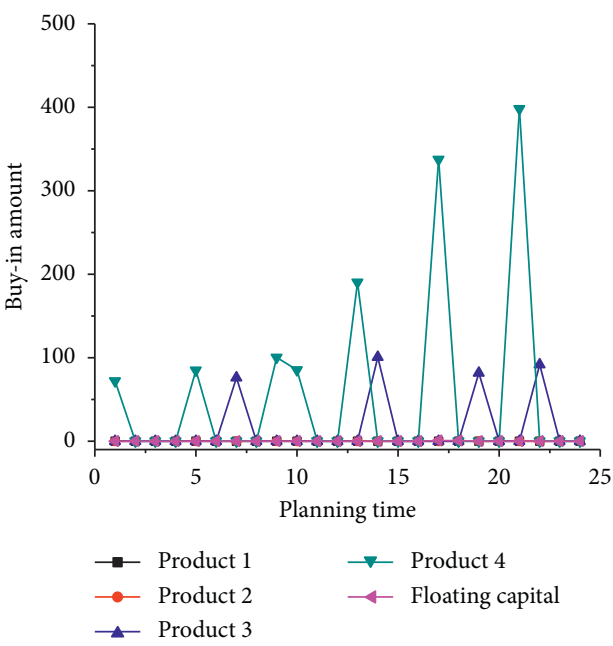

(d)

Figure 9: Continued. 


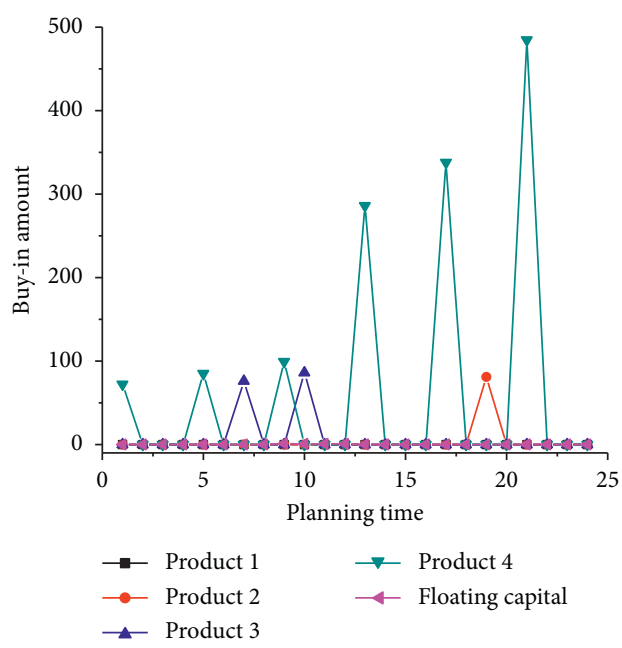

(e)

FiguRE 9: Structures of endpoints with maximum return with different $\underline{c}$ for set $_{1}$ instance $_{2}$ : (a) $\underline{c}=2$, (b) $\underline{c}=4$, (c) $\underline{c}=6$, (d) $\underline{c}=8$, and (e) $\underline{c}=10$.

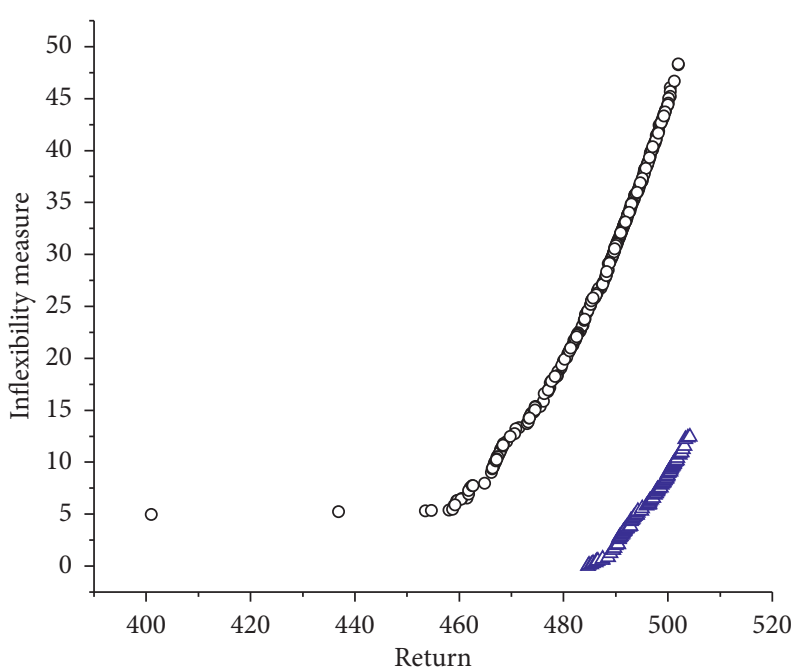

$\triangle \mathrm{SBX}$ crossover

O BLX- 0.5 crossover

(a)

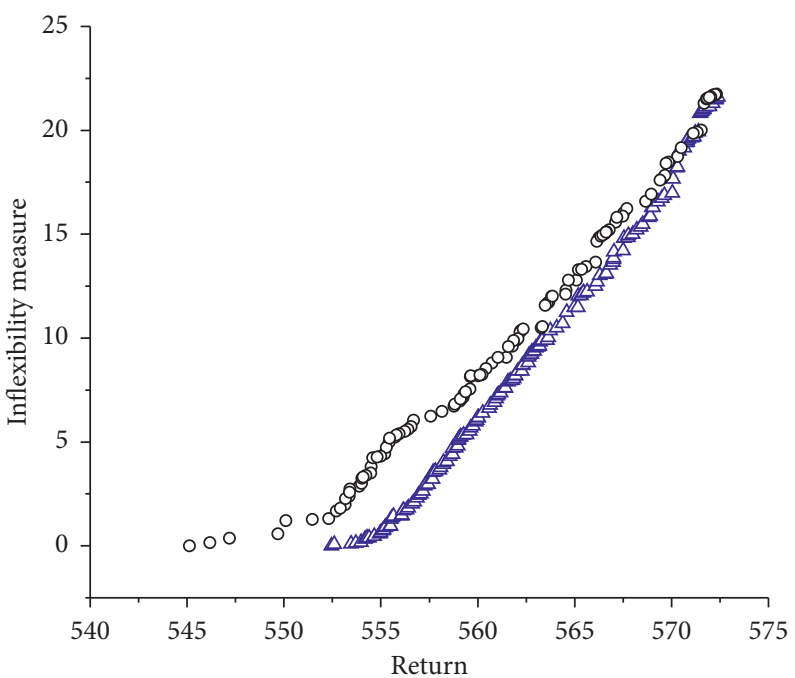

$\triangle$ SBX crossover

O BLX- 0.5 crossover

(b)

FIgURE 10: Nondominated solutions with BLX-0.5 and SBX crossovers: (a) set instance $_{1}$ and (b) set instance $_{2}$.

probability for the real-valued matrix. In the experiments, the crossover probability for the binary matrix $\left(\mathrm{pc}_{\mathrm{bin}}\right)$ and the mutation probabilities $\left(\mathrm{pm}_{\text {real }}, \mathrm{pm}_{\text {bin }}\right)$ were constant and set as $\mathrm{pc}_{\text {bin }}=0.9, \mathrm{pm}_{\text {real }}=0.1$, and $\mathrm{pm}_{\text {bin }}=0.1$. One can see from the two figures that the algorithm performed worst with $\mathrm{pc}_{\text {real }}=0.3$. What should be noted is that a high level of the real-valued matrix crossover probability, $\mathrm{pc}_{\text {real }}=0.9$, as suggested in other studies, could not achieve the best performance. While with an intermediate level of $\mathrm{pc}_{\text {real }}=0.7$ or $\mathrm{pc}_{\text {real }}=0.5$, the algorithm performed better. If we look at the average values of hypervolume and IGD in 30 runs, compared with $\mathrm{pc}_{\text {real }}=0.5$, the performance with $\mathrm{pc}_{\text {real }}=0.7$ shows some advantages for the first 4 instances. However, for set $_{2}$ instance $_{5}$ and set $_{2}$ instance $_{6}$, the algorithm with $\mathrm{pc}_{\text {real }}=0.5$ performed better than $\mathrm{pc}_{\text {real }}=0.7$. This difference may be derived from the scale of the instance. For large scale instances, a relatively lower level of $\mathrm{pc}_{\text {real }}$ might be more effective. Then, in the following experiments, we fixed $\mathrm{pc}_{\text {real }}=0.7$ for set $_{1}$ instance $_{1}$ to set ${ }_{2}$ instance $_{4}$ and $\mathrm{pc}_{\text {real }}=$ 0.5 for set $_{2}$ instance $_{5}$ and set $_{2}$ instance $_{6}$. We then analyzed the sensitivity of $\mathrm{pc}_{\mathrm{bin}}$ with constant $\mathrm{pc}_{\text {real }}$. Box charts of hypervolume and IGD values are reported in Figures 13 and 14 , respectively. One can see that, for most instances, the algorithm with $\mathrm{pc}_{\mathrm{bin}}=0.9$ performed best. Only for set $_{2}$-instance ${ }_{4}$, the algorithm performed best with pc $c_{\text {bin }}=0.7$ and the performance was followed by the algorithm with 


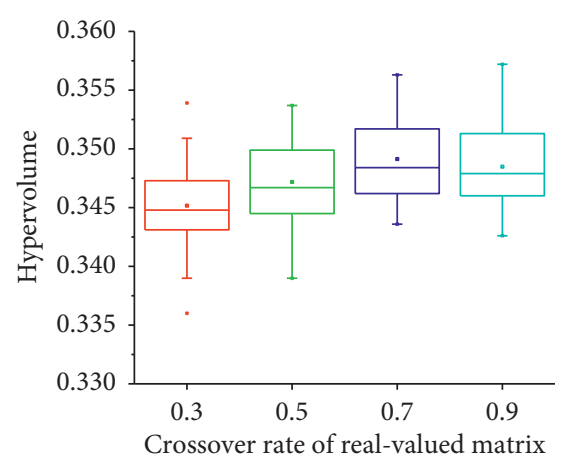

(a)

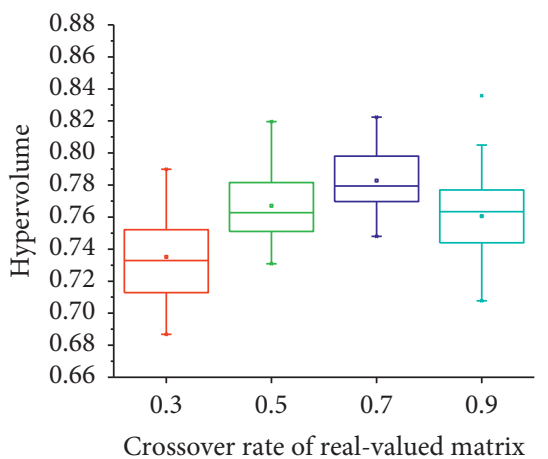

(d)

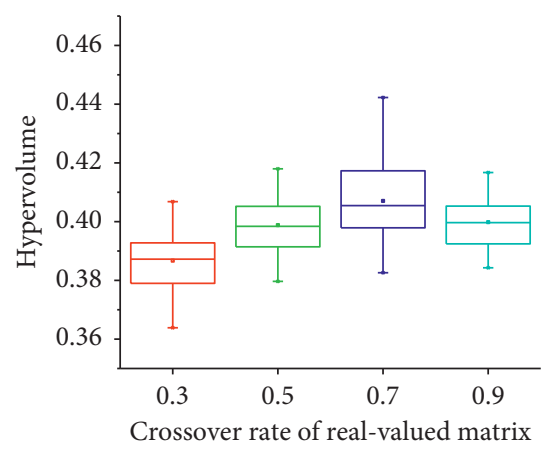

(b)

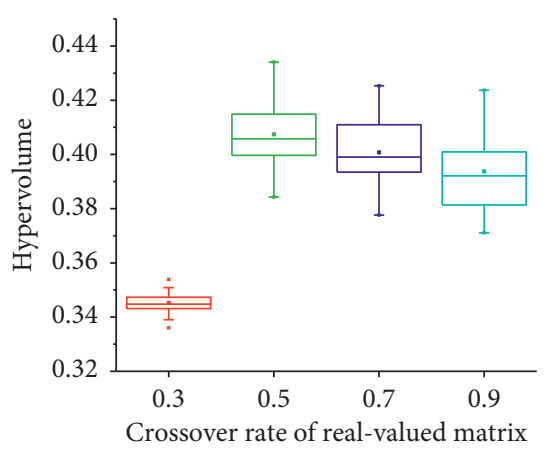

(e)

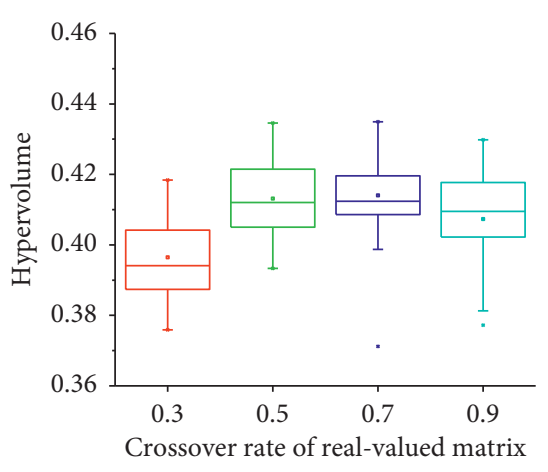

(c)

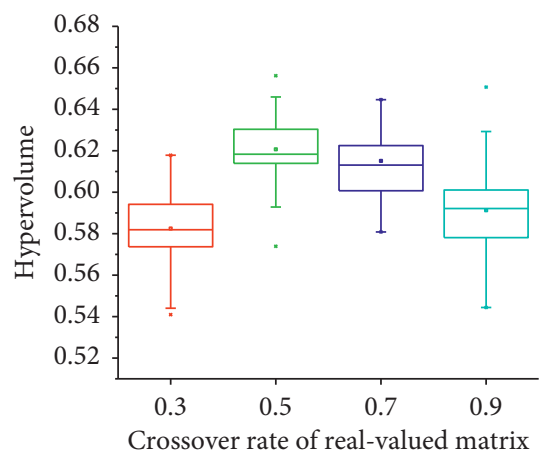

(f)

FIGURE 11: Box charts of hypervolume on set $_{2}$ with different levels of crossover probability for real-valued matrix $\left(p c_{\text {bin }}=0.9\right)$ : $(a)$ set $_{2}$ instance $_{1}$, (b) set $_{2}$ instance $_{2}$, (c) set $_{2}$ instance $_{3}$, (d) set $_{2}$ instance $_{4}$, (e) set $_{2}$ instance $_{5}$, and (f) set $_{2}$ instance $_{6}$.

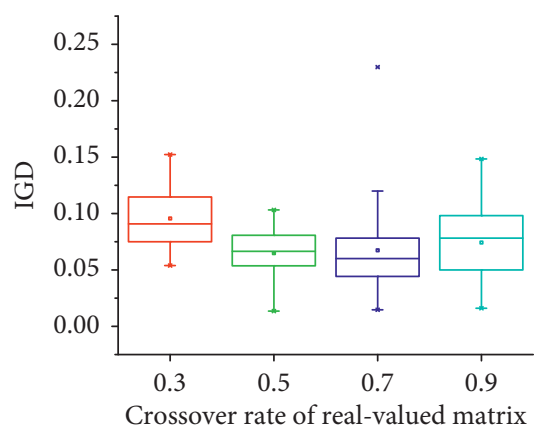

(a)

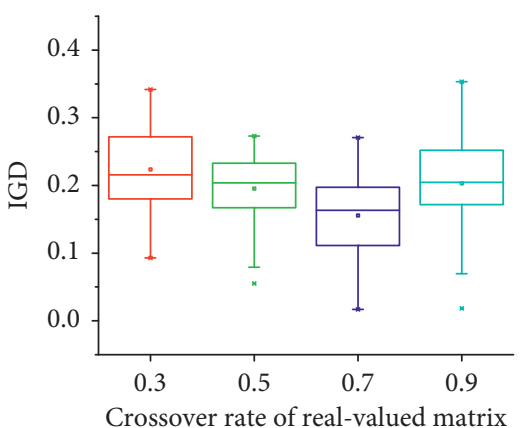

(d)

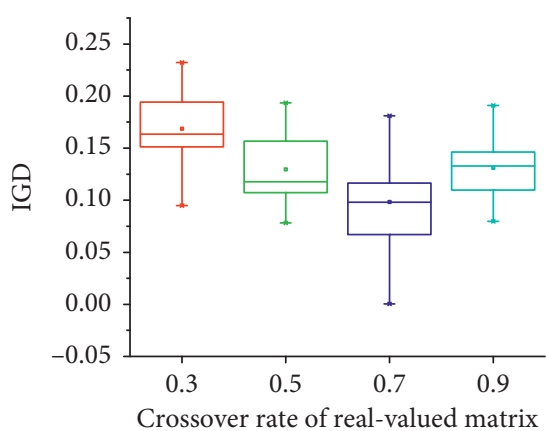

(b)

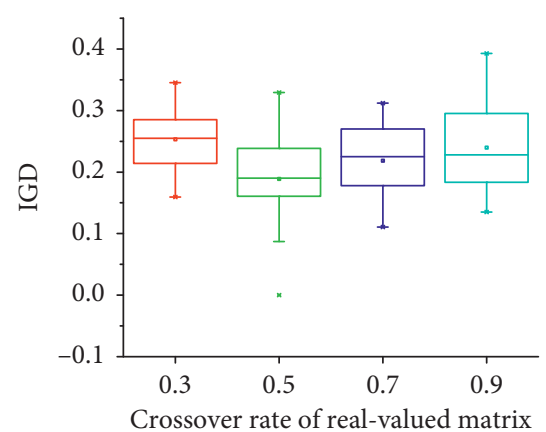

(e)

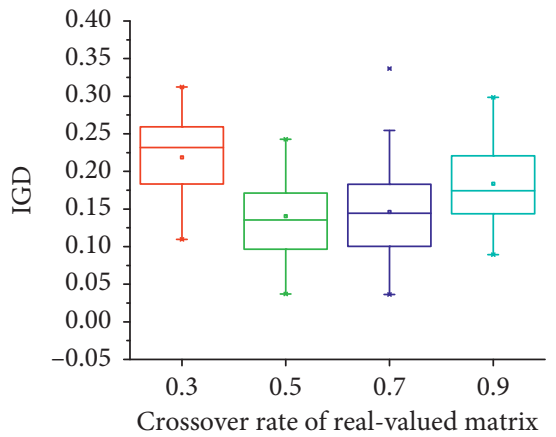

(c)

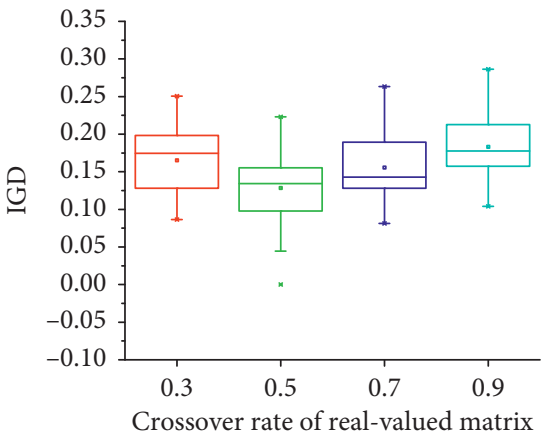

(f)

FIGURE 12: Box charts of IGD on set ${ }_{2}$ with different levels of crossover probability for real-valued matrix ( $\left.\mathrm{p}_{\mathrm{bin}_{\text {in }}}=0.9\right)$ : (a) set $_{2}$ instance $_{1}$, (b) set $_{2}$ instance $_{2}$, (c) set ${ }_{2}$ instance $_{3}$, (d) set $_{2}$ instance $_{4}$, (e) set ${ }_{2}$ instance $_{5}$, and (f) set $_{2}$ instance $_{6}$. 


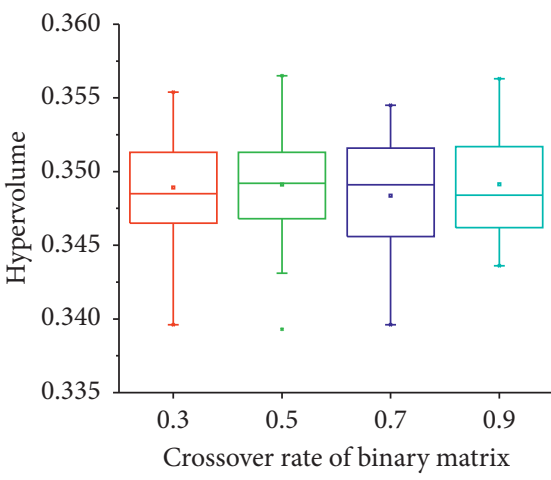

(a)

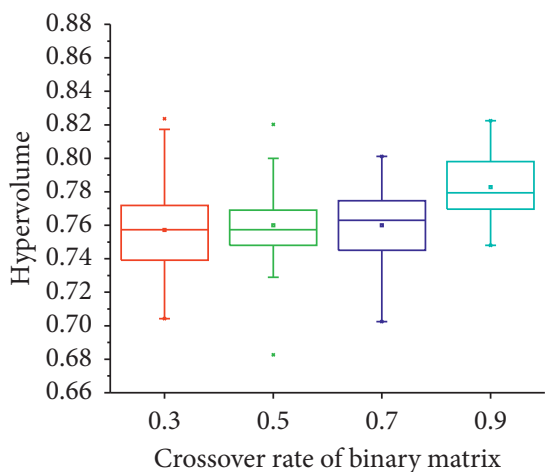

(d)

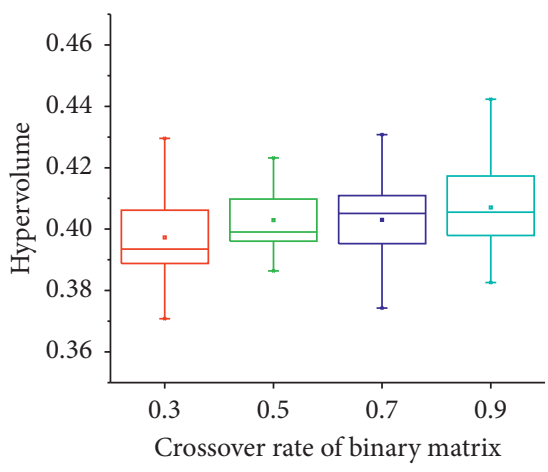

(b)

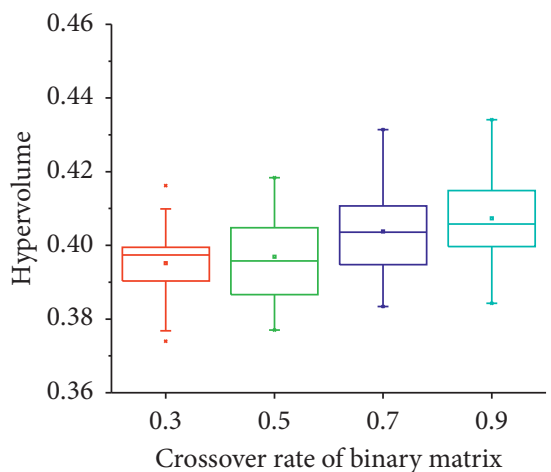

(e)

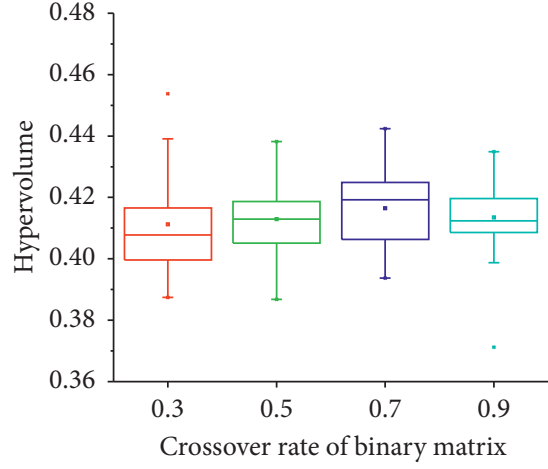

(c)

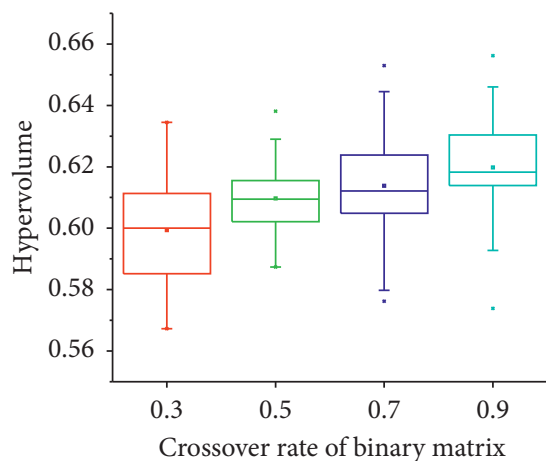

(f)

Figure 13: Box charts of hypervolume on set $_{2}$ with different levels of crossover probability for binary matrix and constant pc $c_{\text {real }}$ : (a) set $_{2 \_}$instance $_{1}$, (b) set $_{2}$ instance $_{2}$, (c) set $_{2}$ instance $_{3}$, (d) set $_{2}$ instance $_{4}$, (e) set $_{2}$ instance $_{5}$, and (f) set instance $_{6}$.

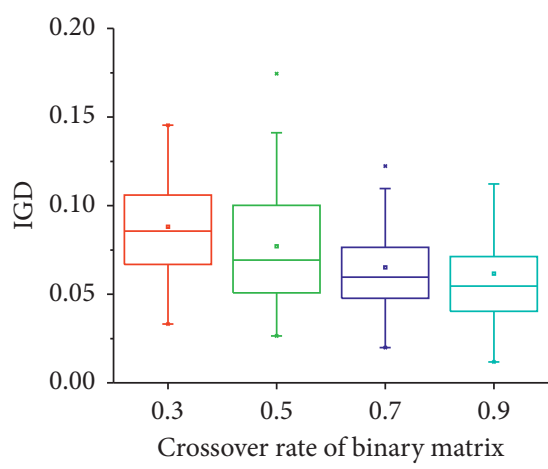

(a)

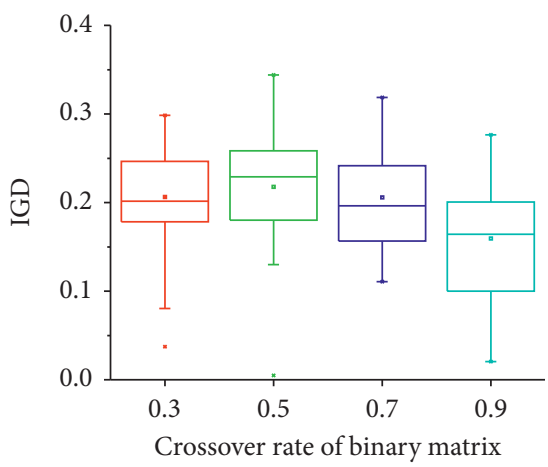

(d)

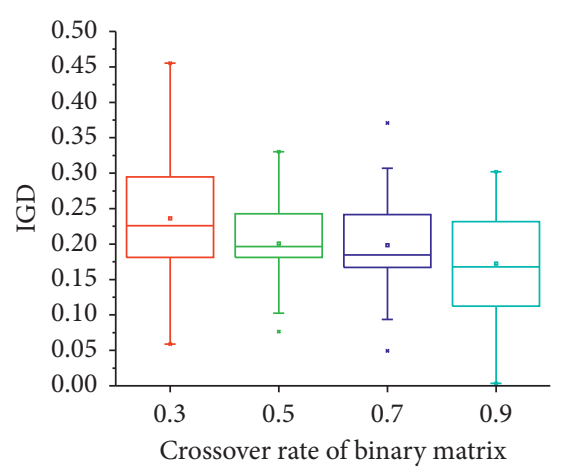

(b)

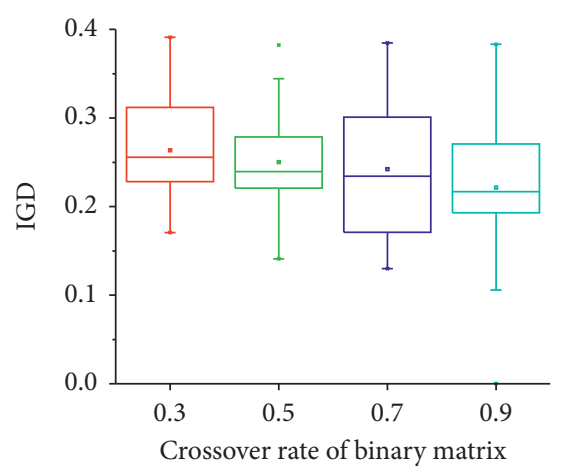

(e)

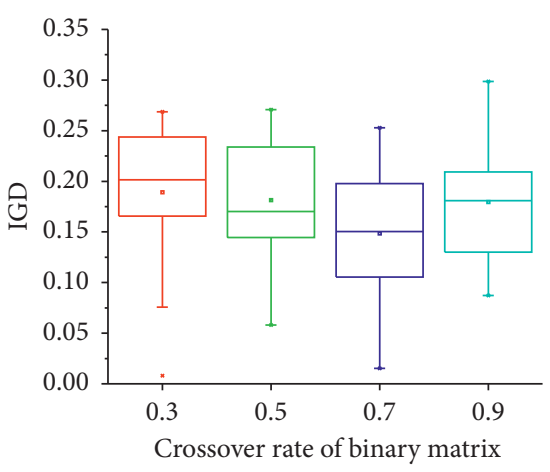

(c)

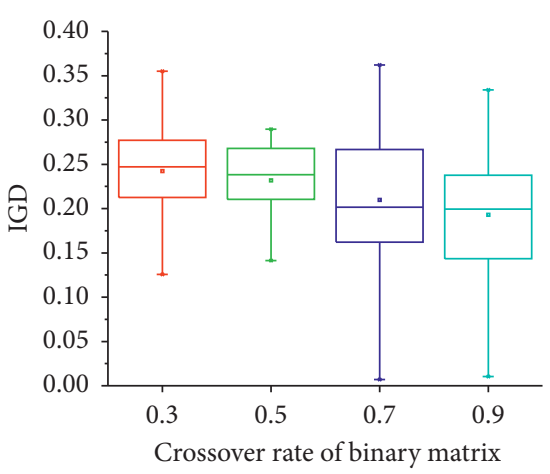

(f)

Figure 14: Box charts of IGD on set ${ }_{2}$ with different levels of crossover probability for binary matrix and constant pc $\mathrm{real}_{\text {: }}$ : (a) set ${ }_{2}$ instance $_{1}$,

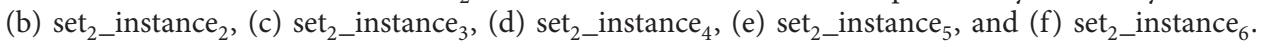




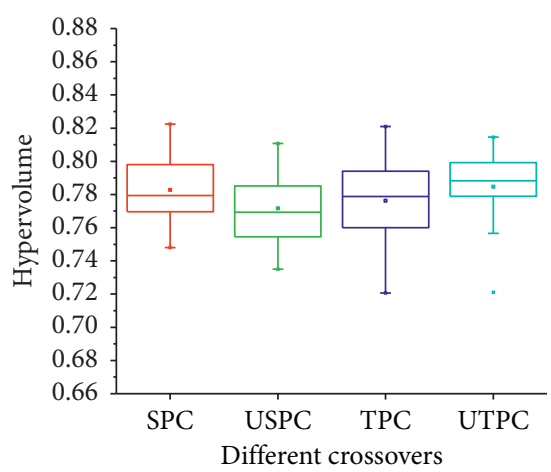

(a)

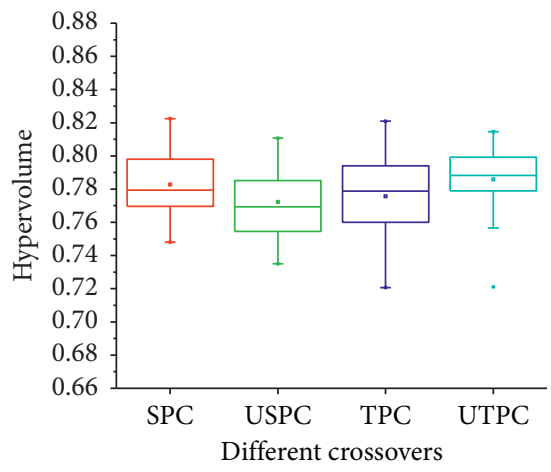

(d)

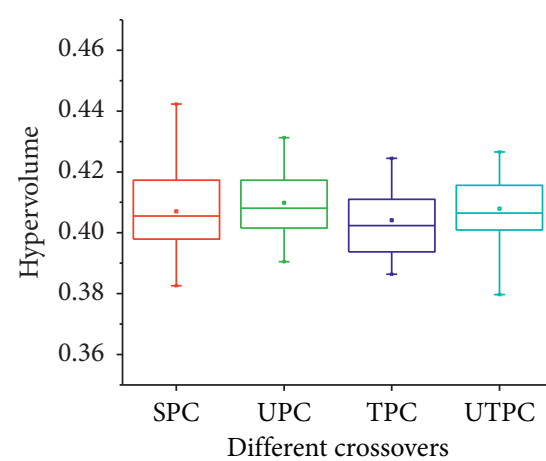

(b)

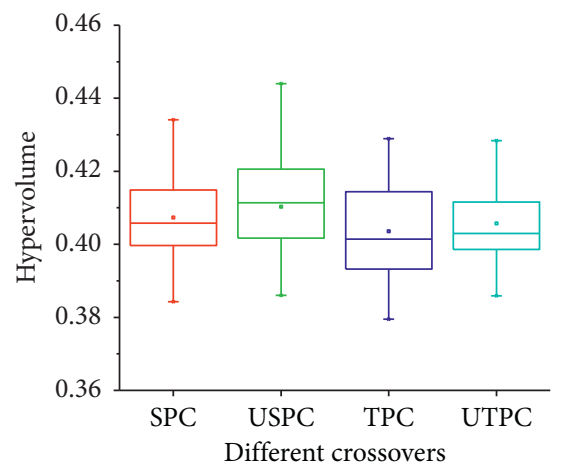

(e)

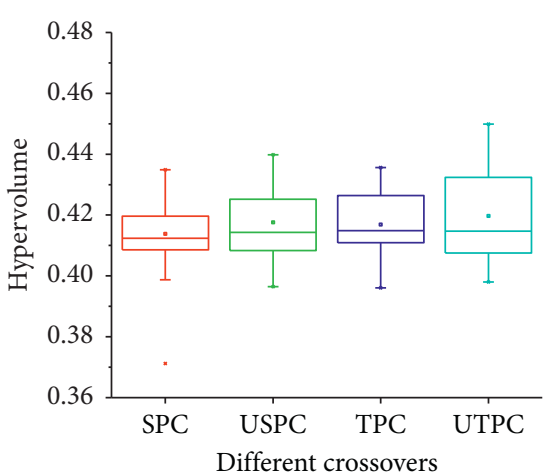

(c)

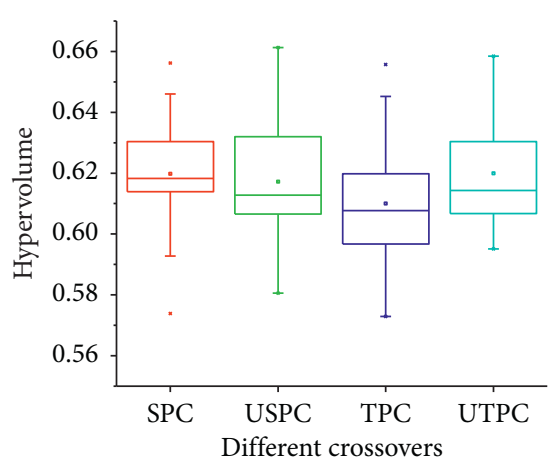

(f)

FIGURE 15: Box charts of hypervolume with different crossovers: (a) set $_{2}$ instance $_{1}$, (b) set ${ }_{2}$ instance $_{2}$, (c) set $_{2}$ instance $_{3}$, (d) set instance $_{4}$, (e) set $_{2}$ instance ${ }_{5}$, and (f) set $_{2}$ instance $_{6}$.

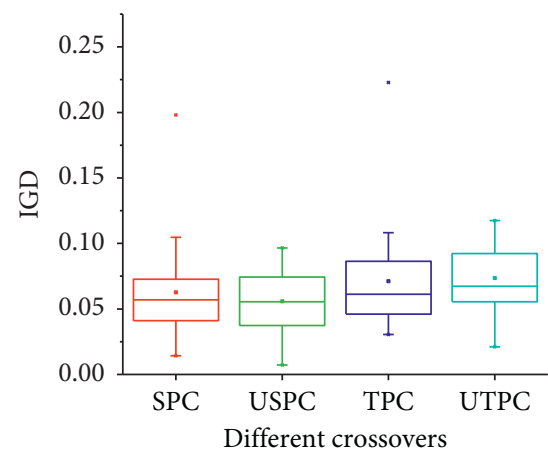

(a)

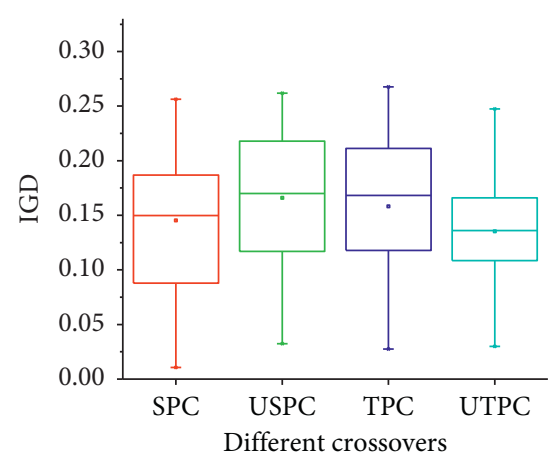

(d)

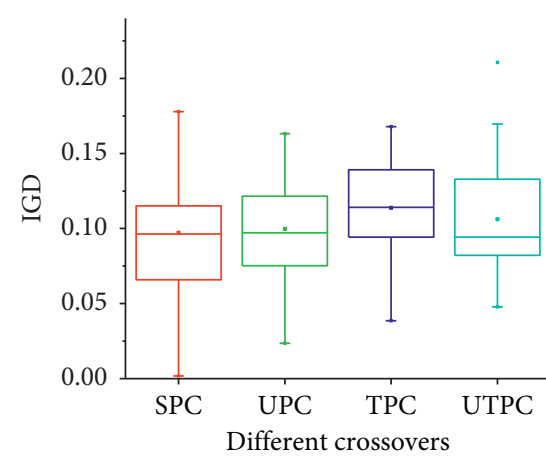

(b)

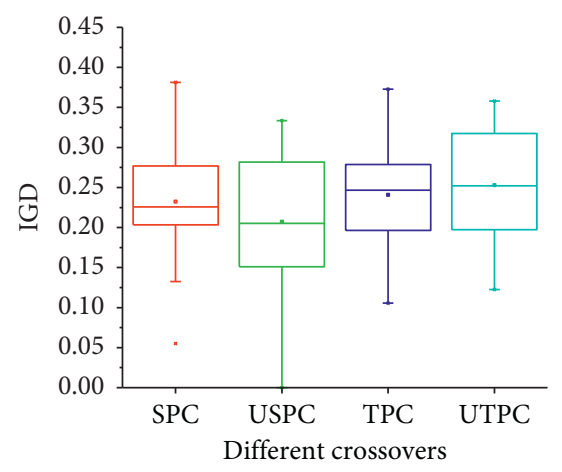

(e)

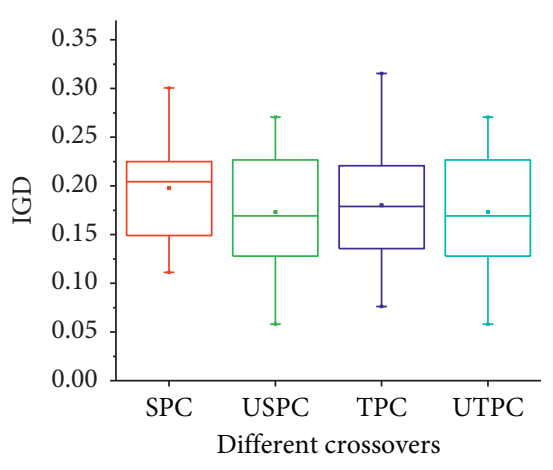

(c)

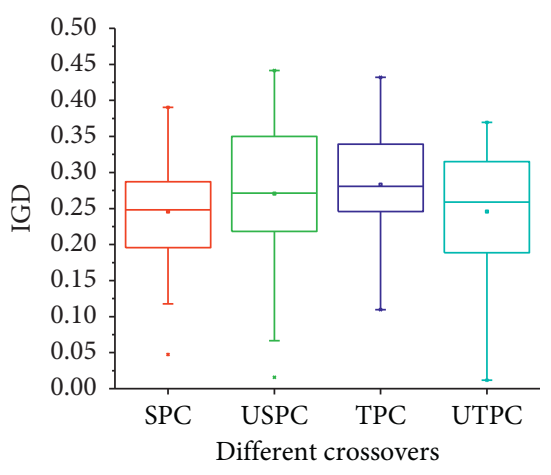

(f)

FIGURE 16: Box charts of IGD with different crossovers: (a) set ${ }_{2}$ instance $_{1}$, (b) set $_{2}$ instance $_{2}$, (c) set ${ }_{2}$ instance $_{3}$, (d) set instance $_{4}$, (e) set $_{2-}$ instance $_{5}$, and (f) set $_{2}$ instance ${ }_{6}$. 


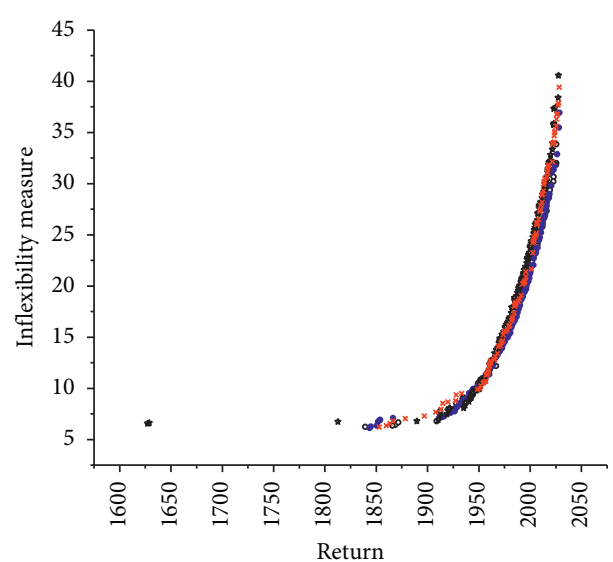

- Single point crossover * Two-point crossover

- Uniform single point $\times$ Uniform two-point crossover

(a)

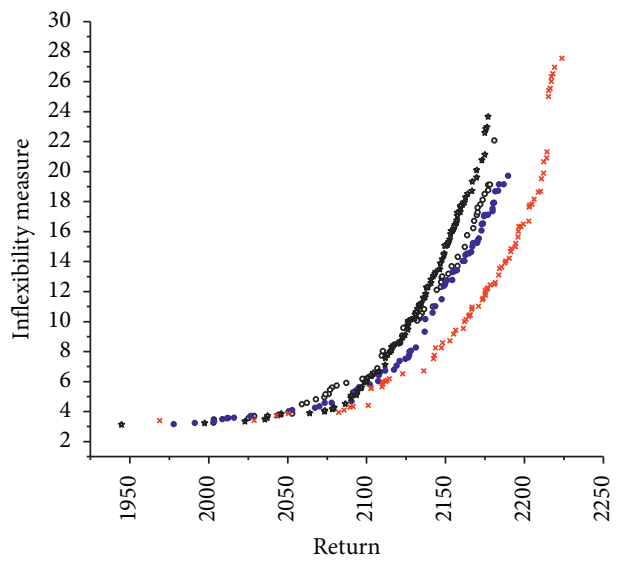

- Single point crossover * Two-point crossover

- Uniform single point $\times$ Uniform two-point crossover

(c)

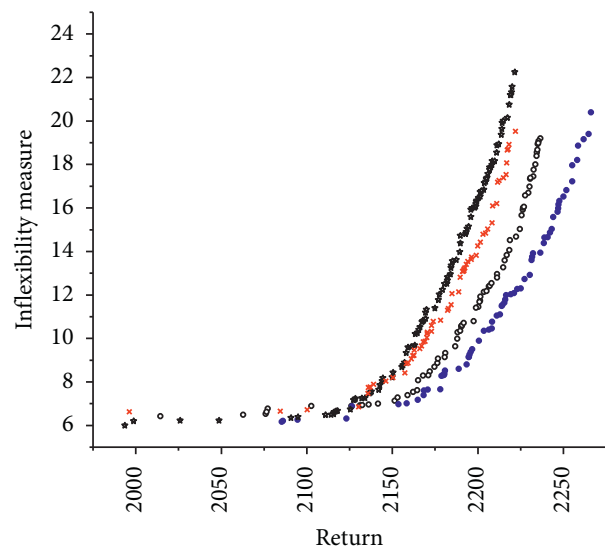

- Single point crossover * Two-point crossover

- Uniform single point $\times$ Uniform two-point crossover

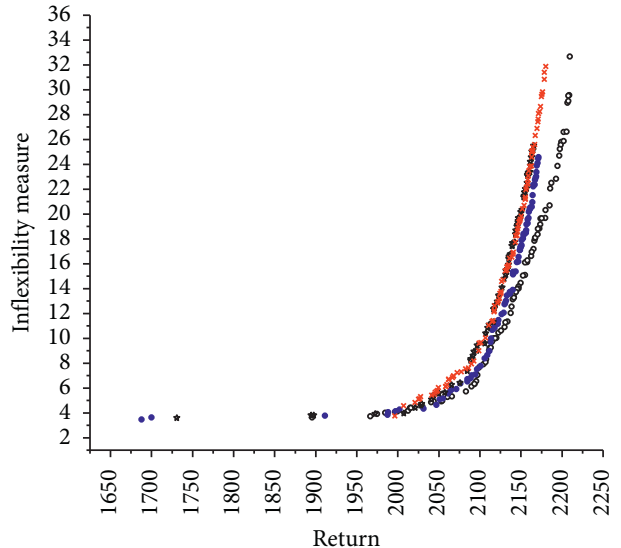

- Single point crossover * Two-point crossover

- Uniform single point $\times$ Uniform two-point crossover

(b)

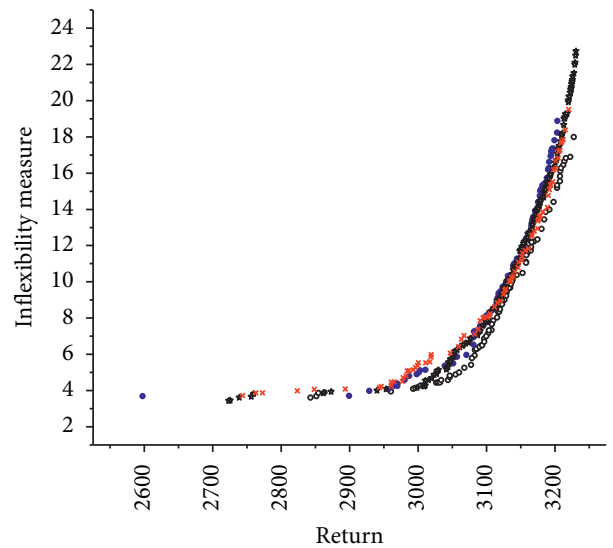

- Single point crossover * Two-point crossover

- Uniform single point $\times$ Uniform two-point crossover

(d)

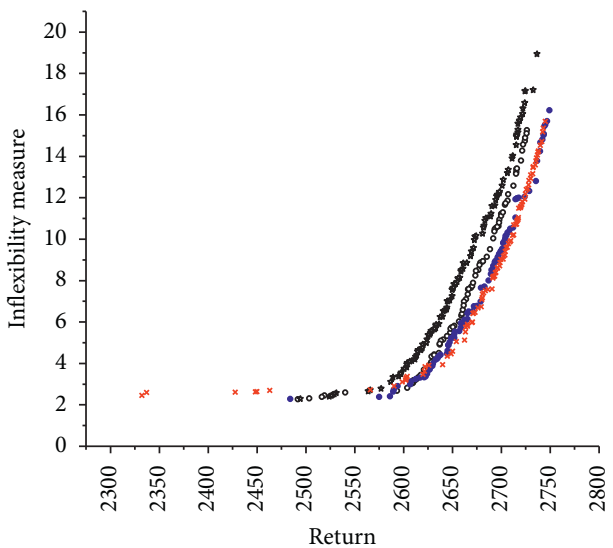

- Single point crossover * Two-point crossover

- Uniform single point $\times$ Uniform two-point crossover

(f)

FIGURE 17: Obtained nondomination solutions in the objective space with different ${\text { crossovers: (a) } \text { set }_{2} \text { instance }_{1} \text {, (b) } \text { set }_{2} \text { instance }}_{2}$, (c) set $_{2}$ instance $_{3}$, (d) set $_{2}$ instance $_{4}$, (e) set $_{2}$ instance $_{5}$, and (f) set $_{2}$ instance $_{6}$. 
$\mathrm{pc}_{\text {bin }}=0.9$. Then, we fixed $\mathrm{p} c_{\text {bin }}=0.9$ in the following experiments. It is noteworthy that effective crossover probabilities for real-valued and binary parts of chromosome are not identical. However, in the literature, the crossover probabilities for both parts were usually set to be the same. Based on the abovementioned results, we suggest to independently investigate the crossover mechanism for portfolio problems with a hybrid encoding.

5.4.2. Performance Comparison of Crossover Operators. Finally, we investigated the effectiveness of four types of crossovers for the binary matrix. Metric values of hypervolume and IGD for single-point crossover, uniform single-point crossover, two-point crossover, and uniform twopoint crossover are presented in Figures 15 and 16, respectively. It can be seen from the figures that it is difficult to determine which crossover performed the best for the test instances. In contrary, it can be considered that these four crossovers have no statistically significant differences. However, decision makers concern the obtained nondominated solutions to a great extent. Thus, we plotted the obtained nondominated solutions in the objectives for all six test instances in Figure 17. One can see from the figure that, for set $_{2}$ instance $_{2}$ and set $_{2}$ instance $_{4}$, the algorithm with the single-point crossover obtained a better approximated Pareto front. While uniform crossovers performed better for set ${ }_{2}$ instance $_{4}$, set ${ }_{2}$ instance $_{5}$, and set ${ }_{2}$ instance $_{6}$. The four crossovers had no remarkable difference for set $_{2}$ instance ${ }_{1}$. From the abovementioned results, we may cautiously suggest the usage of uniform crossover for largescale problems. It can be seen that the mechanism of the crossover for binary matrix affects the quality of obtained nondominated solutions to a large degree. Thus, in future research studies, it is worthwhile to investigate the designing of more effective crossover mechanisms for the addressed problem.

\section{Conclusions}

In this paper, we address the problem of portfolio management of bank financial products with a long-term planning horizon. Instead of considering portfolio risk, we concentrate on the flexibility of a portfolio. A multiobjective optimization model with objectives of portfolio return and flexibility is presented. The proposed model is also characterized by multiperiod investment where capital is available step by step and the decision maker needs to decide her investment behavior at each decision point. Due to the nonconvex and discrete characteristics of the problem, a multiobjective evolutionary algorithm is presented as the solution technique. As suggested in the literature, a hybrid binary/real-valued chromosome is employed for encoding. In the experimental analysis, two sets of test instances are randomly generated for illustrating the addressed problem and testing the proposed approach. Experimental results of parameter sensitivity suggest that crossovers on different parts of the chromosome should be separately considered. Performance comparison of different crossovers show that the effective crossover mechanism for the problem is still a quite open issue. Additionally, phenotype analysis is presented based on two small size instances.

The presented model and solution technique proposed in this paper provides risk-averse investors with an appropriate decision support tool. When the investors make a long-term financial planning, they can trade off the final return and the flexibility of a portfolio. However, we are aware of that some issues need to be considered in the model, such as unexpected changes of financial products, uncertainty of return rates, and dynamic or incremental decision in the long run. We will focus on these topics in future research studies.

\section{Data Availability}

The data used to support the findings of this study are available from the corresponding author upon request.

\section{Conflicts of Interest}

The authors declare that there are no conflicts of interest regarding the publication of this paper.

\section{Acknowledgments}

This work was supported by the National Natural Science Foundation of China under Grant nos. 71501181, 71871185, 71910107002, and U1811462.

\section{References}

[1] H. Markowitz, "Portfolio selection," The Journal of Finance, vol. 7, no. 1, pp. 77-91, 1952.

[2] H. Markowitz, Mean-Variance Analysis in Portfolio Choice and Capital Markets, Blackwell, Oxford, UK, 2001.

[3] P.-C. Lin and P.-C. Ko, "Portfolio value-at-risk forecasting with ga-based extreme value theory," Expert Systems with Applications, vol. 36, no. 2, pp. 2503-2512, 2009.

[4] H. Konno and H. Yamazaki, "Mean-absolute deviation portfolio optimization model and its applications to Tokyo stock market," Management Science, vol. 37, no. 5, pp. 519531, 1991.

[5] M. Mallikarjuna and R. P. Rao, "Evaluation of forecasting methods from selected stock market returns," Financial Innovation, vol. 5, no. 1, p. 40, 2019.

[6] R. Gopinathan and S. R. S. Durai, "Stock market and macroeconomic variables: new evidence from India," Financial Innovation, vol. 5, no. 1, p. 29, 2019.

[7] M. R. Galankashi, F. M. Rafiei, and M. Ghezelbash, "Portfolio selection: a fuzzy-anp approach," Financial Innovation, vol. 6, no. 1, p. 17, 2020.

[8] A. Joshi, S. Pradhan, and J. P. Bist, "Savings, investment, and growth in Nepal: an empirical analysis," Financial Innovation, vol. 5, no. 1, p. 39, 2019.

[9] A. Nazaritehrani and B. Mashali, "Development of e-banking channels and market share in developing countries," Financial Innovation, vol. 6, no. 1, p. 12, 2020.

[10] L. Chen, J. Peng, B. Zhang, and I. Rosyida, "Diversified models for portfolio selection based on uncertain semivariance," International Journal of Systems Science, vol. 48, no. 3, pp. 637-648, 2017. 
[11] M. B. Kar, S. Majumder, S. Kar, and T. Pal, "Cross-entropy based multi-objective uncertain portfolio selection problem," Journal of Intelligent \& Fuzzy Systems, vol. 32, no. 6, pp. 4467-4483, 2017.

[12] S. Majumder, S. Kar, and T. Pal, "Mean-entropy model of uncertain portfolio selection problem," Multi-Objective Optimization: Evolutionary to Hybrid Framework, Springer, Singapore, 2018.

[13] S. Majumder, P. Kundu, S. Kar, and T. Pal, "Uncertain multiobjective multi-item fixed charge solid transportation problem with budget constraint," Soft Computing, vol. 23, no. 10, pp. 3279-3301, 2019.

[14] S. Arnone, A. Loraschi, and A. Tettamanzi, "A genetic approach to portfolio selection," Neural Network World, vol. 3, pp. 597-604, 1993.

[15] D. Maringer and H. Kellerer, "Optimization of cardinality constrained portfolios with a hybrid local search algorithm," OR Spectrum, vol. 25, no. 4, pp. 481-495, 2003.

[16] F. Streichert, H. Ulmer, and A. Zell, "Evaluating a hybrid encoding and three crossover operators on the constrained portfolio selection problem," in Proceedings of the 2004 Congress on Evolutionary Computation (IEEE Cat. No. 04TH8753), pp. 932-939, Portland, OR, USA, June 2004.

[17] F. Streichert, H. Ulmer, and A. Zell, "Comparing discrete and continuous genotypes on the constrained portfolio selection problem," in Genetic and Evolutionary Computation-GECCO 2004, pp. 1239-1250, Springer, Berlin, Geramany, 2004a.

[18] T. Cura, "Particle swarm optimization approach to portfolio optimization," Nonlinear Analysis: Real World Applications, vol. 10, no. 4, pp. 2396-2406, 2009.

[19] G. A. V. Pai and T. Michel, "Evolutionary optimization of constrained $k$-means clustered assets for diversification in small portfolios," IEEE Transactions on Evolutionary Computation, vol. 13, no. 5, pp. 1030-1053, 2009.

[20] R. Ruiz-Torrubiano and A. Suárez, "A memetic algorithm for cardinality-constrained portfolio optimization with transaction costs," Applied Soft Computing, vol. 36, pp. 125-142, 2015.

[21] S. Guo, L. Yu, X. Li, and S. Kar, "Fuzzy multi-period portfolio selection with different investment horizons," European Journal of Operational Research, vol. 254, no. 3, pp. 10261035, 2016.

[22] K. Doerner, W. J. Gutjahr, R. F. Hartl, C. Strauss, and C. Stummer, "Pareto ant colony optimization: a metaheuristic approach to multiobjective portfolio selection," Annals of Operations Research, vol. 131, no. 1-4, pp. 79-99, 2004.

[23] J. Branke, B. Scheckenbach, M. Stein, K. Deb, and H. Schmeck, "Portfolio optimization with an envelope-based multi-objective evolutionary algorithm," European Journal of Operational Research, vol. 199, no. 3, pp. 684-693, 2009.

[24] K. Metaxiotis and K. Liagkouras, "Multiobjective evolutionary algorithms for portfolio management: a comprehensive literature review," Expert Systems with Applications, vol. 39, no. 14, pp. 11685-11698, 2012.

[25] A. Ponsich, A. L. Jaimes, and C. A. C. Coello, "A survey on multiobjective evolutionary algorithms for the solution of the portfolio optimization problem and other finance and economics applications," IEEE Transactions on Evolutionary Computation, vol. 17, no. 3, pp. 321-344, 2013.

[26] S. K. Mishra, G. Panda, and R. Majhi, "A comparative performance assessment of a set of multiobjective algorithms for constrained portfolio assets selection," Swarm and Evolutionary Computation, vol. 16, pp. 38-51, 2014.
[27] M. B. Kar, S. Kar, S. Guo, X. Li, and S. Majumder, "A new biobjective fuzzy portfolio selection model and its solution through evolutionary algorithms," Soft Computing, vol. 23, no. 12, pp. 4367-4381, 2019.

[28] K. Liagkouras, "A new three-dimensional encoding multiobjective evolutionary algorithm with application to the portfolio optimization problem," Knowledge-Based Systems, vol. 163, pp. 186-203, 2019.

[29] M. Woodside-Oriakhi, C. Lucas, and J. E. Beasley, "Portfolio rebalancing with an investment horizon and transaction costs," Omega, vol. 41, no. 2, pp. 406-420, 2013.

[30] S. S. Meghwani and M. Thakur, "Multi-objective heuristic algorithms for practical portfolio optimization and rebalancing with transaction cost," Applied Soft Computing, vol. 67, pp. 865-894, 2018.

[31] W. J. Gutjahr, S. Katzensteiner, P. Reiter, C. Stummer, and M. Denk, "Multi-objective decision analysis for competenceoriented project portfolio selection," European Journal of Operational Research, vol. 205, no. 3, pp. 670-679, 2010.

[32] X. Cui, J. Gao, X. Li, and D. Li, "Optimal multi-period meanvariance policy under no-shorting constraint," European Journal of Operational Research, vol. 234, no. 2, pp. 459-468, 2014.

[33] A. K. Sethi and S. P. Sethi, "Flexibility in manufacturing: a survey," International Journal of Flexible Manufacturing Systems, vol. 2, pp. 289-328, 1990.

[34] J. Xiong, L.-N. Xing, and Y.-W. Chen, "Robust scheduling for multi-objective flexible job-shop problems with random machine breakdowns," International Journal of Production Economics, vol. 141, no. 1, pp. 112-126, 2013.

[35] J. Xiong, J. Liu, Y. Chen, and H. A. Abbass, "A knowledgebased evolutionary multi-objective approach for stochastic extended resource investment project scheduling problems," IEEE Transactions on Evolutionary Computation, vol. 18, no. 5, pp. 742-763, 2014.

[36] J. Xiong, R. Leus, Z. Yang, and H. A. Abbass, "Evolutionary multi-objective resource allocation and scheduling in the Chinese navigation satellite system project," European Journal of Operational Research, vol. 251, no. 2, pp. 662-675, 2016.

[37] J. Xiong, R. Wang, and J. Jiang, "Weapon selection and planning problems using MOEA/D with distance-based divided neighborhoods," Complexity, vol. 2019, Article ID 7589760, 18 pages, 2019.

[38] K. Deb, A. Pratap, S. Agarwal, and T. Meyarivan, "A fast and elitist multiobjective genetic algorithm: nsga-II," IEEE Transactions on Evolutionary Computation, vol. 6, no. 2, pp. 182-197, 2002.

[39] N. Kozodoi, S. Lessmann, K. Papakonstantinou, Y. Gatsoulis, and B. Baesens, "A multi-objective approach for profit-driven feature selection in credit scoring," Decision Support Systems, vol. 120, pp. 106-117, 2019.

[40] I. Yevseyeva, E. B. Lenselink, A. de Vries, A. P. IJzerman, A. H. Deutz, and M. T. M. Emmerich, "Application of portfolio optimization to drug discovery," Information Sciences, vol. 475, pp. 29-43, 2019.

[41] E. Zitzler and L. Thiele, "Multiobjective evolutionary algorithms: a comparative case study and the strength Pareto approach," IEEE Transactions on Evolutionary Computation, vol. 3, no. 4, pp. 257-271, 1999.

[42] D. A. V. Veldhuizen and G. B. Lamont, "Multiobjective evolutionary algorithms: analyzing the state-of-the-art," Evolutionary Computation, vol. 8, no. 2, pp. 125-147, 2000. 